\title{
LncRNA SNHG16 Facilitates Liver Metastases of Colorectal Cancer by Modulating Circulating Tumor Cells (CTCs) Epithelial- Mesenchymal Transition (EMT) via a Positive Feedback Loop with YAP1/TEAD1 Complex
}

\author{
Zhenxian Xiang \\ Wuhan University Second Clinical Hospital: Wuhan University Zhongnan Hospital \\ Guoquan Huang \\ Department of Gastrointestinal Surgery \\ Haitao Wu \\ Wuhan University Second Clinical Hospital: Wuhan University Zhongnan Hospital \\ Qiuming He \\ Wuhan University Second Clinical Hospital: Wuhan University Zhongnan Hospital \\ Chaogang Yang \\ Wuhan University Second Clinical Hospital: Wuhan University Zhongnan Hospital \\ Rongzhang Dou \\ Wuhan University Second Clinical Hospital: Wuhan University Zhongnan Hospital \\ Qing Liu \\ Wuhan University Second Clinical Hospital: Wuhan University Zhongnan Hospital \\ Jialing Song \\ Wuhan University Second Clinical Hospital: Wuhan University Zhongnan Hospital \\ Yan Fang \\ Wuhan University Second Clinical Hospital: Wuhan University Zhongnan Hospital \\ Shuyi Wang \\ Wuhan University Second Clinical Hospital: Wuhan University Zhongnan Hospital \\ bin Xiong ( $\sim$ binxiong1961@whu.edu.cn) \\ Wuhan University Zhongnan Hospital
}

Research

Keywords: SNHG16, miR-195-5p, YAP1, TEAD1, CTCs, cancer

Posted Date: December 29th, 2020

DOI: https://doi.org/10.21203/rs.3.rs-135600/v1

License: @ (i) This work is licensed under a Creative Commons Attribution 4.0 International License. Read Full License 


\section{Abstract}

Background: Circulating tumor cells are important precursor of colorectal cancer metastasis, which attributes to the main cause of cancer-related death. The ability to adopt epithelial-mesenchymal transition (EMT) process facilitates CTCs generation, thereby overcoming metastatic bottlenecks and realizing distant metastasis. However, the potential molecular mechanism of CRC EMT remains largely unknown.

Methods: RT-qPCR, immunohistochemical staining, and western blot were used to detect the expression of mRNA and protein in CRC. Loss- and gain-of-function approaches were performed to investigate the effect of SNHG16 on CRC cell phenotypes. Function assays, including wounding healing, transwell assay, and clone formation were used to assess the effect of SNHG16 on tumor biological behavior. Then, RNA immunoprecipitation, Chromatin Immunoprecipitation, Co-Immunoprecipitation, GST-pull down, biotin-labeled miR195-5p pull down, and dual-luciferase assay were performed to uncover the underlying mechanism for molecular interaction. Finally, CRC nude mice xenograft model experiment was performed to evaluate the influence of SNHG16 on tumor progression in vivo

Results: Compared with normal tissue and cell line, SNHG16 was significantly upregulated in CRC. Clinical investigation revealed that SNHG16 high expression was correlated with advanced TNM stage, distant metastasis, and poor prognosis of cancer patients. According to Loss- and gain-of-function experiment, SNHG16 could promote CRC proliferation, migration, invasion, EMT, mesenchymaltype CTCs ( $\left.{ }^{\mathrm{M}} \mathrm{CTCs}\right)$ generation, and liver metastasis through YAP1 in vitro and in vivo. Mechanistic research indicates that, SNHG16 could act as miRNA sponge to sequester miR-195-5p on Ago2, thereby protecting YAP1 from repression and facilitating CRC liver metastasis and tumor progression. Moreover, YAP1 could combine with TEA Domain Transcription Factor 1 (TEAD1) to form a YAP1/TEAD1 complex, which could in turn bind to the promoter of SNHG16 and regulate its transcription. In addition, both of YAP1 and TEAD1 are indispensable during this process. Finally, we demonstrated that YAP1 significantly promoted the tumor progression, and SNHG16 could rescue the effect of YAP1 on tumor progression

Conclusion: Herein, we clarified a hitherto unexplored positive feedback loop between SNHG16 and YAP1/TEAD1. These findings provided new sights in CRC liver metastasis, and it may act as a potential candidate in the treatment of CRC.

\section{Background}

Colorectal cancer (CRC) is the third most common malignancy and the second leading cause of cancer-related mortality worldwide ${ }^{1}$. Up to now, metastasis remains the leading cause of cancer-related mortality worldwide ${ }^{2}$. Liver is the most common site of CRC distant metastasis $^{3-5}$, and liver metastasis is the main cause of CRC-related death ${ }^{6}$. Metastasis is a complicated process involving multiple steps and driven by multiple mechanisms ${ }^{7-9}$. Epithelial-to-mesenchymal transition (EMT) process, which provided cancer cells with several prometastatic traits ${ }^{10,11}$, are implicated in the metastatic process ${ }^{12}$. Through the process of EMT, cancer cells could penetrate into blood vessels, thereby forming circulating tumor cells (CTCs). Mounting evidence demonstrated that, CTCs are the precursors of metastases ${ }^{13}$. Moreover, epithelial CTCs could gain more mesenchymal traits by EMT program to increase their invasive ability ${ }^{14}$, thereby overcoming metastatic bottlenecks ${ }^{15,16}$ and achieving great potential for metastasis ${ }^{17-20}$. Accumulating evidence revealed that the counts of CTCs, especially mesenchymal-type $\mathrm{CTCs}^{21}$, are correlated with tumor metastasis ${ }^{22,23}$. So, further illuminate the molecular mechanism of EMT will allow us to better understand the mechanisms of CRC liver metastasis.

The activation of the EMT stems from the interplay of many different factors, among which, long noncoding RNAs (IncRNAs) are some of the most hotly debated ${ }^{24}$. LncRNAs have a transcripts longer than 200 bases, and don't have protein-coding potential ${ }^{25}$. In addition, the complicated secondary structure of IncRNAs contains many binding sites, which allows IncRNAs to drive many important tumor cellular phenotype changes through their interactions with other cellular macromolecules including chromatin, protein, and RNA ${ }^{25,26}$. Consequently, IncRNAs could regulate the EMT in a variety of ways and exert vital influence in this process ${ }^{27,28}$. Among many cancerrelated IncRNAs, small nucleolar RNA host gene 16 (SNHG16) is mainly located in cytoplasm and originally identified as oncogene in neuroblastoma ${ }^{29}$. It has been demonstrated that IncRNA-SNHG16 could regulate cancer cellular proliferation, invasion, EMT, and chemoresistance ${ }^{30,31}$; meanwhile, SNHG16 could also regulate migration, and invasion of CRC, thereby playing pivotal roles in cancer metastasis ${ }^{32}$. Although several target modulators of SNHG16 has been reported in other cancers, such as $221^{31}$ and STAT3 ${ }^{33}$, the molecular mechanism underlying the oncogenic functions of SNHG16 in CRC require further exploration. The identification of upstream and downstream targets of SNHG16 would help elucidate its critical role in CRC liver metastasis. 
Currently, accumulating evidence demonstrated that the Hippo signaling pathway plays important roles in cancers' metastasis ${ }^{34-37}$. As a downstream transcriptional coactivator of the Hippo pathway, the abnormal expression of Yes Associated Protein 1 (YAP1) will promote the malignant tumor's proliferation and metastasis, induce EMT and produce possible drug resistance ${ }^{38-40}$. YAP1 in its active form, on the other hand, could function as a transcriptional co-activator predominantly mediated by an interaction with TEAD transcription factors ${ }^{41}$. MiR-195-5p has been demonstrated be a tumor suppressor in various cancers ${ }^{42}$. In a previous study, we also have demonstrated that miR-195-5p could potently suppress the expression of YAP1, and thus inhibit the process of EMT in CRC ${ }^{43}$. Intriguingly, using bioinformatics, we found same miR-195-5p response elements on SNHG16 and YAP1. Therefore, we wonder whether IncRNA-SNHG16 could interplay with miR-195-5p/YAP1 axis and regulate the state of CTCs' EMT, thereby affecting liver metastasis of colorectal cancer. More importantly, exploring the underlying molecular mechanism of the YAP1-IncRNA-microRNA interplay has great significance.

In present study, we found that SNHG16 was upregulated in CRC and significantly associated with poor prognosis of CRC patients. Coxregression analysis revealed that SNHG16 may represent an independent prognostic biomarker in CRC. According to Loss- and gain-of function analysis, YAP1 was the most significant changed EMT-related transcription factors, and SNHG16 could affect tumor progression through YAP1 in vivo and in vitro. Mechanistically, SNHG16 could act as miRNA sponge to sequester miR-195-5p on Ago2, thereby protecting YAP1 from repression and facilitating CRC liver metastasis. Moreover, YAP1 could combine with TEAD1 to form a complex, which in turn bind with the promoter of SNHG16 to regulate its transcription. Herein, we clarified a hitherto unexplored positive feedback loop between SNHG16 and YAP1/TEAD1. These finding provides a novel mechanism for CRC liver metastasis, and it may be a potential candidate in the treatment of CRC.

\section{Method And Material Patient samples}

This study was approved by the Research Ethics Committee of Wuhan University (Wuhan, Hubei, PR China). Informed consents were obtained from all participating patients. The participating patient must be diagnosed with primary CRC by histopathologic diagnosis and undergone surgeries with complete prognostic information. No local or systemic neoadjuvant radiotherapy, or/and chemotherapy and targeted therapy were managed. One-hundred and eleven human CRC tissues and PANT (distance to cancer $>5 \mathrm{~cm}$ ) were randomly obtained from patients in the Zhongnan Hospital of Wuhan University between January 2014 and December 2015. Each sample was snap-frozen in liquid nitrogen and then stored at $-80^{\circ} \mathrm{C}$.

\section{CTC isolation and identification}

The isolation and enrichment of CTC was performed by CTCBIOPSY device (Wuhan YZY Medical Science and Technology Co., Ltd., Wuhan, China), which was described in our previous research ${ }^{44}$. According to the manufacture's instruction, we diluted $1 \mathrm{ml}$ mice blood into $5 \mathrm{ml}$ with $0.9 \%$ sodium chloride solution, the total liquid was then transferred to ISET tubes with $8 \mu \mathrm{m}$ diameter aperture membrane. Through the positive pressure from 12 to $20 \mathrm{mmHg}$ in ISET tubes, candidate CTC was adhered to the membrane of ISET tube and identified by three-color immunofluorescence.

\section{Cell culture and treatment}

Human colon cancer cell lines DLD1, Caco2, SW480, SW620, HT29, lovo, Hct116 and normal intestinal epithelium cell line NCM460 were obtained from the Cell Bank of Wuhan University. Cells were maintained in an incubator at $37^{\circ} \mathrm{C}$ and $5 \% \mathrm{CO}_{2}$. Cells were cultured in DMEM medium (Gibco, USA) Supplemented with $2 \mathrm{mmol} / \mathrm{L}$ glutamine and 10\% fetal calf serum (Gibco, USA).

\section{Transient transfection and stable transfection}

To induce miR-195-5p overexpression and inhibition, cells were transfected with miR-195-5p mimics and inhibitors (RiboBio Co., Ltd) using Lipofectamine 2000. To verify the transfection efficiency, miR-195-5p mimic negative control and miR-195-5p inhibitor negative control were included in each transfection experiment. For SNHG16, a pool of three siRNAs were purchased from genepharma and transfected into cells via lip2000 to select the best knockdown effect.

To construct stable transfection cell lines, anti-SNHG16 Lentiviral vectors (Lv-anti-SNHG16) and their nonspecific Lentiviral vectors (Lvanti-SNHG16 NC) containing green fluorescent protein (GFP) were constructed in the Genepharma (Shanghai, China). In addition, SNHG16-overexpression Lentiviral vectors (Lv-Oe-SNHG16) and their nonspecific Lentiviral vectors (Lv-Oe-SNHG16 NC) containing

Page $3 / 27$ 
Mecherry were purchased from Genecopoeia (Wuhan, China). We also induce the overexpression and inhibition of YAP1 and lentiviral vectors (Lv-Oe-YAP1/ Lv-Oe-YAP1 NC, Lv-anti-YAP1/ Lv-anti-YAP1 NC) in the GeneChem Chemical Technology (Shanghai, China). According to the instruction of manufacture, we get the MOI of HCT116 $(\mathrm{MOI}=10)$ and DLD1 $(\mathrm{MOI}=20)$. For virus infection, virus and polybrene (final $7 \mu \mathrm{g} / \mathrm{ml}$, Sigma Aldrich, Cat\#107689) was added to $25 \%$ confluent cells. Fresh media was added 16 hours after infection. Media was changed with media containing appropriate antibiotics 48 hours after infection. After Puro selection, cells were maintained for at least one day without drug for further experiments.

\section{Isolation of RNA and performance of qRT-PCR}

Total RNA was extracted from tissue samples and cell lines using a the Trizol Reagent (Invitrogen, USA) according to the manufacturer's instructions. Nanodrop2000 was used to quantify the concentration of RNA and $1 \mu \mathrm{g}$ of total RNA was reverse transcribed into cDNA. We performed the cDNA synthesis of mRNA by using Primescript ${ }^{\text {TM }}$ RT reagent Kit (Vazyme, Nanjing, China). cDNA synthesis for microRNA detection was carried out using miRNA 1st Strand cDNA Synthesis Kit (Vazyme, Nanjing, China). cDNA was used for subsequent qRT-PCR using the SYBR-Green PCR Master Mix (Vazyme, Nanjing, China). Each reaction was run on the BioRad IQ5 Real time PCR machine (BioRad, USA). Relative expression was calculated using the 2- $\Delta \triangle \mathrm{Ct}$ method. The primer sequences are listed in Table S1 (supplementary Table 1).

\section{Western blot and antibodies}

Cells were lysed using RIPA buffer supplemented with complete proteinase inhibitor cocktail (Thermo Scientific, USA). Then, the lysate was homogenized using sonication and quantified using BCA reaction. Total proteins were separated by SDS-PAGE and subsequently transferred to PVDF membranes (Millipore, USA). Prior to incubation with diluted primary antibodies and HRP-conjugated secondary antibodies, blots were blocked in non-fat milk for an hour. The primary antibodies and dilution folds are as follows: anti-E-cadherin (1:1000), anti-Vimentin (1:1000; Proteintech, USA), anti-YAP1 (1:1000), anti-GAPDH (1:5000).

\section{Luciferase reporter assay}

To evaluate whether miRNA regulate SNHG16, the wild type SNHG16 and a mutant SNHG16 sequence (mutant in miR-195-5p binding site) were amplified from the genomic DNA and sub-cloned into the pmiR-RB-Report (Promega, USA), we called it h-SNHG16-Wild and hSNHG16-Mut, respectively. To assess whether miR-195-5p regulates YAP1 directly, we constructed wild-type and mutated YAP1 3' -UTR (YAP1-WT/ YAP1-Mut) luciferase reporter vectors in the pmiR-RB-Report (Promega, USA). For the SNHG16 promoter assay, SNHG16 promoter $(-2000 /+1)$ and its construction $(-1861 /+1,-1794 /+1,-1368 /+1)$ were amplified from the genomic DNA and inserted into pGL3-Baisic (Promega, USA). Then the CDNA of SNHG16 promoter containing the predicted TEAD1 binding sites was subcloned into the pGL3-Basic vector (Promega, USA), termed as PGL3-SNHG16-pro. Then, the TEAD1 binding sites mutated SNHG16 promoter was synthesized by RiboBio (RiboBio Co., Ltd), termed as PGL3-SNHG16-pro-mut (1 and 2). Transfection was carried out using lipofectamine 2000 and luciferase activities were determined using Dual-Luciferase reporter system (Promega, USA) according to the manufacturer's instructions.

\section{RNA immunoprecipitation (RIP) assay}

According to the instruction of Magna RIP RNA-Binding Protein Immunoprecipitation Kit (Millipore, Billerica, MA, USA), we performed the RIP to investigate the binding of microRNA-195-5p to IncRNA SNHG16. The anti-Ago2 antibodies (CST, USA) were used for immunoprecipitation, and the species anti-IgG antibodies and total RNA (input controls) were used for control. The co-precipitated RNAs was reverse transcribed to cDNA and detected by qRT-PCR.

\section{Co-immunoprecipitation}

CRC cells were lysed in RIPA buffer and the protein concentration of lysates quantified using BCA reaction. After impurities removed, the clarified lysates was incubated with rabbit anti-YAP1 or anti-TEAD1 for overnight end-over-end shaking at $4{ }^{\circ} \mathrm{C}$. Subsequently, we use the Protein $A / G$ sepharose beads to capture the antigen-antibody complexes in $4{ }^{\circ} \mathrm{C}$ for $12 \mathrm{~h}$. The operation were followed by the wash of beads with PBS, and the complexes were boiled to separate antigens and antibodies from the beads. After elution from the beads, the expression level of YAP1 and TEAD1 in the IP was analyzed by western blot.

\section{Pulldown assay with biotinylated miRNA}

To assay whether miR-195-5p bind to IncRNA SNHG16 or not, a biotin-avidin pulldown system was applied. The biotinylated miR-195$5 p$ was synthesized by RioBio (RiboBio Co., Ltd), we called it miR-195-5p-Bio. Then, the SNHG16 binding sites mutated biotinylated miR-195-5p was also synthesized in RiboBio, termed as miR-195-5p-mut-Bio. We also used the NC-Bio as a negative control to ensure 
the accuracy of the result, and the operation process are as follows. Put it simply, CRC cells were transfected with biotinylated miR-195$5 p$ by Lipofectamine 2000 , and cells were rinsed and lysed in a buffer 48 hours later. Then, the cell lysates were treatment with streptaviden magnetic beads. Then, The bound RNAs were isolated by TRIzol LS reagent for further RT-PCR analysis.

ChIP

We performed ChIP using the SimpleChIP® Enzymatic Chromatin IP Kit (ChIP) Kit (Cell Signaling, \#9003, USA), as per the instruction of manufacturer. Put simply, after the cells were crosslinked and lysed, the cells become lysates. Subsequently, the lysates were sonicated into 300 bp to 600 bp fragments and immunoprecipitated by anti-TEAD1 antibody (Abcam). Besides, we used Normal rabbit immunoglobulin $\mathrm{G}(\mathrm{IgG})$ as a negative control. Then, we performed the reverse crosslinking and DNA purification. Finally, the precipitated DNA was analyzed by qRT-PCR via the SYBR-Green PCR Master Mix (Vazyme, Nanjing, China). Primers used for SNHG16 promoter regions were shown in supplementary material.

\section{Gst-pull down}

To test whether YAP1 could interact with TEAD1 directly, we performed the GST pull-down assay as follows. First, after the transformant, cloning and expression of GST-YAP1 fusion protein in Escherichia coli, GST-tagged YAP1 fusion protein was further purification using glutathione-Sepharose 4B beads. For pull-down assay, the GST or GST-tagged YAP1 fusion protein were incubated with purified His tagged TEAD1 from Escherichia coli for $4-8 \mathrm{~h}$ in $4^{\circ} \mathrm{C}$. Then, the protein bound glutathione-Sepharose 4B beads were washed four times and the proteins were recovered. Finally, the recovered proteins were analyzed by SDS-PAGE.

\section{Colony formation and wound healing assay}

For the colony formation detection, HCT116 were placed in 6-well plates at a density of 1000 cells per well. In the same way, DLD1 were placed at a density of 1000 cells per well. After two week's culture in incubator, the cells were fixed with $4 \%$ paraformaldehyde and stained with $0.5 \%$ crystal violet. A wound-healing assay was also used to measure the migratory capacity of CRC cells. Cells were seeded at 6-well plate and allowed to grow until the confluence reached by $90 \%$. The 20 ul pipette tip was used to scrap several lines across the cell surface, and then plates were washed three times to remove non-adherent debris. Finally, the cells were incubated at $37^{\circ} \mathrm{C}$ with serum-free medium and photos were taken per 12 hours.

\section{Transwell migration and invasion assay}

24-well Transwell ( $8 \mu \mathrm{m}$ pore size; Corning, USA) was used to assess the cell migration and invasion ability of CRC cells. For migration assay, $1.0 \times 10^{5}$ cells in $0.4 \mathrm{ml}$ serum-free DMEM medium were seed on the upper surface of the well. For invasion assay, transwell chambers were coated with Matrigel gel and $1.5 \times 10^{5}$ cells were seeded on the upper surface of the chamber in $0.4 \mathrm{ml}$ serum-free medium. After $48 \mathrm{~h}$ of incubation, cells on the upper surface of chamber were removed by cotton swabs, the remaining cells on the lower surface of chamber were fixed and stained with $0.5 \%$ crystal violet solution.

\section{Xenograft assays}

The animal experiment were approved by Zhongnan hospital of Wuhan University ethical committee, and performed in accordance with the Guide for the Care and Use of Laboratory Animals of Wuhan University. To perform tumor proliferation assay, 12 BALB/c nude mice (4-6 weeks old) (Hubei Research Center of Laboratory Animals, China) were randomly divided into three groups ( $n=4$ per group): NC (HCT116), Lv-anti-SNHG16 (HCT116), Lv-anti-SNHG16/ Lv-Oe-YAP1 (HCT116). In total, $5 \times 10^{6}$ HCT116 cells suspended in 200 ul medium were subcutaneously injected into the right flank of mice. After ten days, tumor size was measured by digital Vernier calipers every 1 week, and the volume of tumor was calculated by the following formula: volume $=1 / 2 x\left(\right.$ length $x$ width $\left.{ }^{2}\right)$. After thirty days, the transplantable tumor and $1 \mathrm{ml}$ mice blood was collected for further research. For metastasis experiment, the mice was randomly divided into three groups ( $n=6$ per group). The stable cell line were injected into the mice via tail vein, and the metastasis was assessed 30 days after injection by dissection. Finally, the mice were euthanized and necropsied to assess metastatic burden. Then, we further examined the tumor tissues, liver and lung tissues by H\&E, IHC staining and RT-PCR assay.

\section{Statistical analysis}

All the data derived from at least three times independent experiment, and p values $<0.05$ were considered statistically significant. Through the SPSS statistical software and GraphPad Prism software, all the statistical analyses were performed. To assess the association between SNHG16 expression and YAP1, and miR-195-5p expression, we used the Pearson's correlation analysis. Then, we applied Chi-square test to analysis the expression of SNHG16 and clinicopathological status of CRC patients. Comparisons between 2

Page 5/27 
groups were performed using 2-tailed, unpaired student's t test. In addition, Kaplan-Meier survival curve and log-rank test was used for survival analysis. Finally, univariate and multivariate Cox-regression analyses were applied to identify the independent factors of prognosis.

\section{Result}

\section{SNHG16 is upregulated in colorectal cancer tissues and indicates poor prognosis of colorectal cancer patients}

Initially we measured the expression of IncRNA-SNHG16 in 45 matched normal colorectal and cancerous colorectal tissues via qRTPCR. Compared with paired adjacent normal tissue (PANT), IncRNA-SNHG16 was significantly upregulated in CRC tissues (Fig. 1A) and correlated with advanced TNM stage.

We then measured the expression level of $111 \mathrm{CRC}$ tissues and separated the samples into two groups based on the median value of expression level. To explore whether the expression of IncRNA-SNHG16 correlated with CRC progression, we investigated the correlation between IncRNA-SNHG16 expression and clinicopathological significance (Table 1). Evidently, the high expression of IncRNA-SNHG16 was positively correlated with lymphovascular invasion (LVI), and perineural invasion (PNI), TNM stage $(p<0.001)$, lymph node metastasis $(p<0.05)$, and distant metastasis $(p<0.001)$, according to the American Joint Committee on Cancer (AJCC) staging system $(p<0.05)$. Further Kaplan-Meier survival analysis revealed that, patients with higher IncRNA-SNHG16 had shorter overall survival (OS) $(p<0.05)$ (Fig. 1C) and progression-free survival (PFS) $(p<0.05)$ (Fig. 1D) than those with lower IncRNA-SNHG16 levels. Meanwhile, cox regression analysis (Table 2) revealed that SNHG16 expression was an independent prognostic factor correlating with poor OS (HR = $3.125,95 \% \mathrm{Cl}=1.145-8.47$ ). Similarly, we found IncRNA-SNHG16 was significantly upregulated in seven CRC cell lines (Fig. 1E); meanwhile, we detected the highest expression level of SNHG16 in HCT116 and the lowest expression of SNHG16 in DLD1, and thus these two cell lines were selected for further research. Additionally, ISH showed that SNHG16 was mainly located in the cytoplasm of CRC tumor cells, but not in PANT (Fig. 1F). Taken together, these results strongly indicated that SNHG16 upregulated in CRC and was an independent prognostic factor. 
Table 1

Correlation between the expression of SNHG16 and clinicopathologic characteristics in CRC

\begin{tabular}{|c|c|c|c|c|}
\hline \multirow[t]{2}{*}{ Paramaters } & \multirow[t]{2}{*}{$\mathbf{n}$} & \multicolumn{2}{|c|}{ SNHG16 expression } & \multirow[t]{2}{*}{$\mathbf{p}$} \\
\hline & & Low & High & \\
\hline \multicolumn{5}{|l|}{ Gender } \\
\hline Male & 63 & 30 & 33 & \multirow[t]{2}{*}{0.784} \\
\hline Female & 48 & 25 & 23 & \\
\hline \multicolumn{5}{|l|}{ Age, years } \\
\hline$<60$ & 48 & 27 & 21 & \multirow[t]{2}{*}{0.298} \\
\hline$>60$ & 63 & 28 & 35 & \\
\hline \multicolumn{5}{|l|}{ Tumor site } \\
\hline$<4$ & 58 & 31 & 27 & \multirow[t]{2}{*}{0.503} \\
\hline$>4$ & 53 & 24 & 29 & \\
\hline \multicolumn{5}{|l|}{ Tumor size, cm } \\
\hline Colon & 56 & 26 & 30 & \multirow[t]{2}{*}{0.636} \\
\hline Rectal & 55 & 29 & 26 & \\
\hline \multicolumn{5}{|c|}{ Tumor differentiation } \\
\hline Moderate/well & 81 & 42 & 39 & \multirow[t]{2}{*}{0.523} \\
\hline Poor & 30 & 13 & 17 & \\
\hline \multicolumn{5}{|l|}{ LVI } \\
\hline Absence & 59 & 38 & 21 & \multirow[t]{2}{*}{0.002} \\
\hline Presence & 52 & 17 & 35 & \\
\hline \multicolumn{5}{|l|}{ PNI } \\
\hline Presence & 56 & 18 & 38 & \multirow[t]{2}{*}{0.001} \\
\hline Absence & 55 & 37 & 18 & \\
\hline \multicolumn{5}{|l|}{ TI } \\
\hline T1-2 & 15 & 11 & 4 & \multirow[t]{2}{*}{0.056} \\
\hline T3-4 & 96 & 44 & 52 & \\
\hline \multicolumn{5}{|l|}{ LNM } \\
\hline No-1 & 86 & 52 & 34 & \multirow[t]{2}{*}{0.003} \\
\hline N2-M3 & 25 & 3 & 22 & \\
\hline \multicolumn{5}{|l|}{ TNM stage } \\
\hline $\mathrm{I} / \mathrm{II}$ & 57 & 48 & 9 & \multirow[t]{2}{*}{0.001} \\
\hline III/IV & 54 & 7 & 47 & \\
\hline \multicolumn{5}{|l|}{ CA199 } \\
\hline$<27$ & 77 & 42 & 35 & \multirow[t]{2}{*}{0.15} \\
\hline$>27$ & 34 & 13 & 21 & \\
\hline
\end{tabular}




\begin{tabular}{|lllll|}
\hline Paramaters & $\mathbf{n}$ & \multicolumn{2}{c|}{ SNHG16 expression } & $\mathbf{p}$ \\
\cline { 3 - 4 } & & Low & High & \\
\hline CEA & & & & \\
\hline$<5$ & 59 & 35 & 24 & $\mathbf{0 . 0 3 7}$ \\
\hline$>5$ & 52 & 20 & 32 & \\
\hline M & & & & \multirow{2}{*}{0.001} \\
\hline No metastasis & 101 & 55 & 46 & \\
\hline Metastasis & 10 & 0 & 10 & \\
\hline Overall & 111 & 55 & 56 & \\
\hline
\end{tabular}


Table 2

Univariate and multivariate analyses of clinicopathologic parameters associated with progression-free survival and overall survival

\begin{tabular}{|c|c|c|c|c|c|c|c|c|c|c|c|c|}
\hline \multirow[t]{3}{*}{ parameters } & \multicolumn{5}{|c|}{ overall survival } & \multicolumn{7}{|c|}{ Progression-free survival } \\
\hline & \multicolumn{3}{|c|}{ univariate analysis } & \multicolumn{2}{|c|}{$\begin{array}{l}\text { Multivariate } \\
\text { analysis }\end{array}$} & \multicolumn{3}{|c|}{ univariate analysis } & \multirow[b]{2}{*}{$\mathbf{P}$} & \multicolumn{3}{|c|}{ Multivariate analysis } \\
\hline & HR & $95 \% \mathrm{Cl}$ & $\mathbf{P}$ & HR & $95 \% \mathrm{Cl}$ & $\mathbf{P}$ & HR & $95 \% \mathrm{Cl}$ & & HR & $95 \% \mathrm{Cl}$ & $\mathbf{P}$ \\
\hline $\begin{array}{l}\text { Gender } \\
\text { Female vs } \\
\text { Male }\end{array}$ & 1.19 & $\begin{array}{l}0.625- \\
2.69\end{array}$ & 0.756 & & & & 1.076 & $0.624,1.856$ & 0.792 & & & \\
\hline $\begin{array}{l}\text { CA19-9 } \\
<27 \text { vs }>27\end{array}$ & 0.806 & $\begin{array}{l}0.404- \\
1.607\end{array}$ & 0.54 & & & & 0.666 & $0.377,1.177$ & 0.162 & & & \\
\hline $\begin{array}{l}\text { CEA (low vs } \\
\text { high) }\end{array}$ & 0.731 & $\begin{array}{l}0.382- \\
1.397\end{array}$ & 0.343 & & & & 0.872 & $\begin{array}{l}0.505 \\
1.507\end{array}$ & 0.624 & & & \\
\hline $\begin{array}{l}\text { PNI (low vs } \\
\text { high) }\end{array}$ & 0.738 & $\begin{array}{l}0.387- \\
1.408\end{array}$ & 0.229 & & & & 0.625 & $0.363,1.074$ & 0.259 & & & \\
\hline $\begin{array}{l}\text { LNM (low vs } \\
\text { high) }\end{array}$ & 0.225 & $\begin{array}{l}0.115- \\
0.443\end{array}$ & 0 & 0.51 & $\begin{array}{l}0.224- \\
1.143\end{array}$ & 0.06 & 0.32 & $0.173,0.591$ & 0.001 & 0.819 & $\begin{array}{l}0.399- \\
1.681\end{array}$ & 0.586 \\
\hline $\begin{array}{l}\text { Tumor size } \\
(<4 \mathrm{~cm} \text { vs }> \\
4 \mathrm{~cm})\end{array}$ & 0.9 & $\begin{array}{l}0.472- \\
1.715\end{array}$ & 0.748 & & & & 0.587 & $0.338,1.018$ & 0.058 & & & \\
\hline $\begin{array}{l}\text { Tumor site } \\
\text { (rectal vs } \\
\text { colon) }\end{array}$ & 1.683 & $\begin{array}{l}0.879- \\
3.224\end{array}$ & 0.135 & & & & 1.657 & $0.962,2.854$ & 0.069 & & & \\
\hline $\begin{array}{l}\text { Age } \\
(<60 \text { vs }>60)\end{array}$ & 0.702 & $\begin{array}{l}0.44- \\
1.344\end{array}$ & 0.286 & & & & 1.076 & $0.624,1.856$ & 0.792 & & & \\
\hline $\begin{array}{l}\text { TI (T1-2 vs } \\
\text { T3-4) }\end{array}$ & 0.443 & $\begin{array}{l}0.157- \\
1.254\end{array}$ & 0.125 & & & & 0.31 & $0.123,0.783$ & 0.013 & 0.417 & $\begin{array}{l}0.163- \\
1.064\end{array}$ & 0.067 \\
\hline $\begin{array}{l}\text { Tumor } \\
\text { differentiation } \\
\text { poor/well vs } \\
\text { moderate }\end{array}$ & 1.18 & $\begin{array}{l}0.583- \\
2.390\end{array}$ & 0.645 & & & & 1.243 & $0.691,2.236$ & 0.467 & & & \\
\hline $\begin{array}{l}\text { Metastasis } \\
\text { absence vs } \\
\text { presence }\end{array}$ & 0.031 & $\begin{array}{l}0.011- \\
0.086\end{array}$ & 0.001 & 0.03 & $\begin{array}{l}0.009- \\
0.102\end{array}$ & 0.01 & 0.02 & $0.005,0.077$ & 0.000 & 0.028 & $\begin{array}{l}0.007- \\
0.113\end{array}$ & 0.000 \\
\hline $\begin{array}{l}\text { TNM stage } \\
\text { I-II vs III-IV }\end{array}$ & 0.226 & $\begin{array}{l}0.110- \\
0.463\end{array}$ & 0.002 & 0.19 & $\begin{array}{l}0.064- \\
0.564\end{array}$ & 0.01 & 0.26 & $0.142,0.458$ & 0.000 & 0.352 & $\begin{array}{l}0.188- \\
0.658\end{array}$ & 0.001 \\
\hline $\begin{array}{l}\text { SNHG16 } \\
\text { Low vs High }\end{array}$ & 0.493 & $\begin{array}{l}0.255- \\
0.953\end{array}$ & 0.045 & 0.32 & $\begin{array}{l}0.118- \\
0.873\end{array}$ & 0.03 & 0.41 & $0.236,0.726$ & 0.002 & 0.535 & $\begin{array}{l}0.226- \\
1.269\end{array}$ & 0.156 \\
\hline $\begin{array}{l}\text { LVI } \\
\text { absence vs } \\
\text { presence }\end{array}$ & 0.327 & $\begin{array}{l}0.169- \\
0.633\end{array}$ & 0.003 & 0.44 & $\begin{array}{l}0.210- \\
0.937\end{array}$ & 0.00 & 0.319 & $0.184,0.555$ & 0.000 & 0.625 & $\begin{array}{l}0.332- \\
1.176\end{array}$ & 0.145 \\
\hline
\end{tabular}

The ectopic expression of IncRNA-SNHG16 affects proliferation, migration, invasion, and EMT of CRC cells

To investigate the role of SNHG16 in tumor progression, the loss- and gain-of-function approaches was applied. First, three sequences of siRNA against SNHG16 were designed and qRT-PCR was used to verify the most efficient sequence (Fig S1A). Thus, the sequence of 
si-SNHG16 (si-3) and its negative control were used to construct the lentiviral-based SNHG16 knockdown stable cell line, termed as Lvanti-SNHG16 and Lv-anti-SNHG16-NC, respectively (Fig. 2A). Then, we constructed a lentiviral-based DLD1 cell line which will stably overexpress SNHG16, termed as Lv-Oe-SNHG16 (Fig. 2B). Finally, the knockdown and overexpression effects were validated by PCR (Fig. 2A and Fig. 2B).

As shown in Fig. 2C, the SNHG16-downregulated HCT116 displayed fewer clones than control group, indicating that the cell proliferation was inhibited by SNHG16 knockdown. In contrast, the clones were much more numerous in the SNHG16 overexpressed DLD1 than their negative control (Fig. 2D). Hence, the overexpression of SNHG16 promoted the proliferation of CRC cells. According to transwell migration assay, the knockdown of SNHG16 significantly reduced the cells crossing the membrane (Fig. 2E), whereas the increased number of cells crossing the membrane occurred after the overexpression of SNHG16 (Fig. 2F). Wound healing assays also revealed the positive effect of IncRNA-SNHG16 on CRC migratory capacity. Evidently, the knockdown of SNHG16 inhibited the migratory speed (Fig. 2G); yet the migratory speed was faster in the SNHG16-overexpressed DLD1 cells than in their control group (Fig. 2H). Furthermore, matrigel-coated transwell invasion assay confirmed the promotive effect of SNHG16 on CRC invasion (Fig. 2E and Fig. 2F).

Given the important role of EMT in CRC cellular migration and invasion ${ }^{45}$, we next investigated whether SNHG16 could induce EMT in CRC cells. Western blot and immunofluorescence revealed that, knockdown of SNHG16 could significantly reduce the expression level of Vimentin but increase the level of E-cadherin in HCT116 (Fig. 2l and Fig. 2J). In contrast, SNHG16 overexpression promoted the expression of mesenchymal markers but inhibited the expression of epithelial markers in DLD1 (Fig. 2I and Fig. 2J).

Altogether, all these results demonstrated that SNHG16 could regulate CRC cellular migration and invasion through affecting the process of EMT.

\section{LncRNA SNHG16 facilitates CRC cellular proliferation, migration, invasion and EMT in a YAP1-dependent manner}

To explore the mechanism by which SNHG16 regulates EMT in CRC cells, we focused on the potential EMT-related transcriptional factor, which could regulate the progression of $\mathrm{CRC}^{46}$. As shown in Fig. $3 \mathrm{~A}$ and $3 \mathrm{~B}$, a panel of transcription factors was screened, and YAP1 showed the most significant change among them. Meanwhile, western blot also revealed that SNHG16 could positively regulate the protein level of YAP1 (Fig. 3C), indicating a potential interaction between SNHG16 and YAP1. In addition, the positive association between the expression of SNHG16 and YAP1 in CRC tissues further demonstrated that YAP1 was the potential target of SNHG16. Thus, YAP1 was selected for further research.

To investigate whether the function of SNHG16 on tumor progression was dependent on YAP1 or not, rescue experiment was performed. The result showed that SNHG16 knockdown could inhibit the proliferation, migration, invasion, and EMT of CRC cells. Evidently, YAP1 overexpression could rescue SNHG16-downregulation-mediated inhibitory effect on proliferation, migration, and invasion (Fig S2A, Fig. 3E, Fig S2C). Meanwhile, SNHG16-overexpression facilitated proliferation, migration, invasion, and EMT of DLD1, and the function of SNHG16-overexpression on tumor progression could be abrogated by YAP1 knockdown (Fig S2B, Fig. 3F, Fig S2D). Immunofluorescence and western blot revealed that the overexpression of SNHG16 could promote the EMT of CRC cells, and the promotive effect on EMT could be rescued by YAP1 knockdown; meanwhile, the SNHG16-knockdown-mediated inhibitory effect on EMT could be rescued by YAP1 overexpression (Fig. 3G and Fig. 3H). These results revealed that YAP1 was functional mediator of SNHG16 in CRC cell lines.

miR-195-5p could potently abrogate the effect of SNHG16/YAP1 axis on tumor progression.

To further investigate how the EMT of CRC cells might be controlled dynamically by SNHG16/YAP1 axis during tumor metastasis, we focused on the involvement of microRNAs (miRNAs) ${ }^{47,48}$. According to the hypothesis of competing endogenous RNAs (ceRNAs), 
certain IncRNAs have many miRNA response elements (MRE) and can function as microRNA sponge to impose a posttranscriptional regulation of target genes in cytoplasm, thus playing an important role in the progression of CRC ${ }^{49,50}$. As shown in Fig. 1C, SNHG16 is mainly located in the cytoplasm, indicating a potential posttranscriptional regulation. Through starBase ${ }^{51}$ and LncBase Predicted ${ }^{52}$, we identified several miRNAs which have potential SNHG16 binding sites, one of which was miR-195-5p. Furthermore, we found the same miR-195-5p response elements between SNHG16 and YAP1 (Fig. 4A), which indicate a potential interplay between these three factors. Thus, miR-195-5p was pursued for detailed research.

We have previously demonstrated that miR-195-5p was markedly downregulated in CRC tissue and cell lines ${ }^{43}$, thus we further investigated the relationship between the expression of SNHG16 and miR-195-5p. The correlation between IncRNA-SNHG16 expression and miR-195-5p was measured in fifty CRC tissues. Significantly, SNHG16 was negative correlated with the expression of miR-195-5p in CRC tissues (two-sided Pearson's correlation, $r=-0.6820, p<0.001$ ) (Fig. 4B). Additionally, miR-195-5p was also significantly decreased after SNHG16 overexpression in DLD1 (Fig. 4C). In contrast, miR-195-5p was drastically increased after knockdown of SNHG16 in HCT116 (Fig. 4D). However, we did not observe significant changes of SNHG16 when we upregulated or downregulated miR-195-5p (Fig. 4E and Fig. 4F).

Next, we tried to investigate whether SNHG16/YAP1 axis exerts its promotive effects on migration, invasion, and EMT progression of CRC cells through miR-195-5p. As illustrated in Fig. 4, overexpression of SNHG16 could promote the proliferation, migration, invasion, and EMT of CRC cells. Evidently, miR-195-5p inhibitor could rescue SNHG16-downregulation-mediated inhibitory effect on proliferation (Fig. 4G), migration (Fig. 4I and Fig. 4K), and invasion of HCT116 (Fig. 4I). In contrast, miR-195-5p mimics could abrogate the enhanced proliferation (Fig. 4H), migration (Fig. $4 \mathrm{~J}$ and $4 \mathrm{~L}$ ), and invasion (Fig. $4 \mathrm{~J}$ ) ability of SNHG16 overexpression. Furthermore, the inhibited EMT of SNHG16 knockdown could be rescued by miR-195-5p inhibitors (Fig. 4M). Meanwhile, miR-195-5p mimics significantly reversed the enhanced EMT of IncRNA SNHG16 overexpression (Fig. 4N). In our previous research, we demonstrated miR-195-5p could also regulate the progression of CRC through YAP1. Based on current and previous research, we successfully demonstrated that miR-195-5p could abrogate the effect of SNHG16/YAP1 axis on tumor progression.

IncRNA-SNHG16 functions as a ceRNA and sponges miR-195-5p, further regulating YAP1 expression and facilitating tumor progression.

Next, we sought to examine whether SNHG16-mediated miR-195-5p regulation occurs through direct binding with miR-195-5p or not. Therefore, RIP, biotin-avidin pulldown, and luciferase assay were applied to conduct further research.

miRNAs are present in the form of miRNA ribonucleoprotein complexes (miRNPs) which contain Argounaute2 (Ago2), the core component of RNA-induced silencing complexes (RISCs) ${ }^{53}$. To validate whether SNHG16 associated with miR-195-5p on Ago2, we performed RNA-binding protein immunoprecipitation (RIP) with Ago2 on HCT116 cells transfected with miR-195-5p inhibitor or transfected with negative control (Fig. 5A). As shown in Fig. 5B, Ago2 protein was successfully immunoprecipitated from HCT116 extracts by Ago2 antibody. Clearly, SNHG16 was significantly decreased in Ago2 immunoprecipitates purified from miR-195-5p inhibitor transfected cells, compared with its level in Ago2 immunoprecipitates purified from their negative control (Fig. 5C). Thus, the RIP assay revealed that SNHG16 was likely in the miR-195-5p-RISC and interacts with miR-195-5p. Additionally, the sequence-specific binding between miR-195-5p and SNHG16 was further validated by a biotin-avidin pulldown system. As Fig. 5D shows, the expression of SNHG16 in miR-195-5p-Bio group was about 30 times higher than that of miR-195-5p-Mut-Bio group or control group. Consistent with our results, the luciferase reporter assays revealed that miR-195-5p significantly inhibited the luciferase activity of SNHG16-WT but not of SNHG16-Mut (Fig. 5E-F). Collectively, these results revealed that SNHG16 could act as miRNA sponge to directly bind with miR-195$5 p$ and exert inhibitory effect on miR-195-5p expression.

As we previously demonstrated ${ }^{43}$, upregulation of miR-195-5p suppresses proliferation, migration, invasion, and EMT of colon cancer cells through targeting the YAP1 mRNA 3'-UTR. Therefore, we wondered whether ectopically expressed SNHG16 prevents miR-195-5p from inhibiting YAP1, thereby affecting the progression of tumor. Through starBase, we found that there existed many same miR-195$5 p$ response elements between SNHG16 and miR-195-5p (Fig. 4A). Thus, we speculated that SNHG16 could regulate the mRNA stability of YAP1 through miR-195-5p. Then, a luciferase assay among SNHG16, miR-195-5p, and YAP1 was performed. Compared with 
SNHG16-mut group, SNHG16 could enhance the luciferase of YAP1-WT, while miR-195-5p could inhibit the luciferase of YAP1-WT. Furthermore, the inhibited luciferase of miR-195-5p group on YAP1-WT could be rescued by SNHG16 (Fig. 5G). We did not find significant changes when we conducted the same experiment on YAP1-mut (Fig. 5G). The luciferase assay among these three factors revealed that, the overexpression of SNHG16 could prevent miR-195-5p from targeting YAP1. Through western blot and qRT-PCR in CRC cell line, we could also found that SNHG16 could positively regulate the expression of YAP1. Furthermore, miR-195-5p could rescue SNHG16-mediated regulation on YAP1 (Fig. 5I-K).

Altogether, SNHG16 could function as miRNA sponge to directly bind and sequester endogenous miR-195-5p, thereby preventing it from inhibiting YAP1 expression and affecting tumor progression.

\section{YAP1 could directly bind with TEAD1, forming a complex that binds to the promoter region of SNHG16 and activates its transcription.}

Since YAP1 functions as an important transcriptional co-activator, the positive correlation between the expression of SNHG16 and YAP1 drew us to investigate the regulatory role of YAP1 in SNHG16 transcription. Then, we constructed a lentiviral-based HCT116 cell line which will stably overexpress or knockdown YAP1, termed as Lv-Oe-YAP1 or Lv-anti-YAP1, respectively. Evidently, overexpression of YAP1 significantly promoted SNHG16 expression (Fig. 6A), whereas knockdown of YAP1 dramatically suppressed the expression level of SNHG16 (Fig. 6B). Furthermore, knockdown of YAP1 could inhibit the proliferation (Fig S3A), migration (Fig S3C and Fig S3E) ,invasion (Fig S3C), and EMT (Fig. 6C) of CRC cell lines. In addition, we observed the promotive effect on tumor progression in Lv-OeYAP1 group (Fig S3B, S3D, S3F, and Fig. 6C).

Although we have demonstrated the important role of YAP1 in SNHG16 expression and tumor progression, the mechanism of YAP1IncRNA interaction remains largely unknown. Based on existing research, YAP1 cannot bind directly with DNA alone. However, YAP1 could combine with transcription factors to regulate the transcription of downstream genes ${ }^{54}$. Among these transcription factors, TEAD1-4 is one of the most common binding molecules ${ }^{55}$. Once bound to TEAD, YAP1 formed a YAP1/TEAD complex to initiate downstream gene transcription ${ }^{54,56}$. Through bioinformatic analysis, We found the potential transcription factors TEAD1. Further study showed that the knockdown of either YAP1 or TEAD1 reduced the expression of SNHG16 (Fig. 6D). And evidently, the inhibitory effect on the expression of SNHG16 was most significant when we simultaneously knocked down both of YAP1 and TEAD1 (Fig. 6D). To investigate whether YAP1 and TEAD1 act synergistically, we overexpressed TEAD1 in the Lv-anti-YAP1 cell line and we did not find significant change on the expression of SNHG16 (Fig. 6D). Similarly, when we overexpressed TEAD1 after YAP1 knockdown, the expression level of SNHG16 was not rescued (Fig. 6D). To further verify the cross-talk between TEAD1 and YAP1, coimmunoprecipitation (co-IP) analysis was applied. The co-IP confirmed that YAP1 could bind to TEAD1 directly or indirectly in HCT116 (Fig. 6E). To further investigate whether YAP1 interact with TEAD1 through directly binding or not, GST-pull down assay was performed. After the GST-tagged YAP1 (Fig. 6F) and His-tagged TEAD1 (Fig. 6G) were successfully induced and purified from Escherichia coli, we incubated these two proteins in $4^{\circ} \mathrm{C}$ for 4-8 h. Finally, western blot was performed to examine the expression of these two proteins. GST-pull down further demonstrated the direct interplay between YAP1 and TEAD1 (Fig. 6H). Collectively, these experiments confirmed that YAP1 could directly interact with TEAD1 and form a complex, which regulated the expression of SNHG16. However, whether YAP1/TEAD1 binds to the promoter of SNHG16 remains largely unclear.

Using JASPAR (http://jaspar.genereg.net) and TRANSFAC (http://transfac.gbf.de/TRANSFAC/), we didn't find possible YAP1 binding sites within 2000 bp upstream of transcriptional start of SNHG16; however, there existed three possible TEAD1 binding sites (Fig. 6I). Therefore, the interplay between YAP1/TEAD1 complex and SNHG16 was selected for further research. Significantly, dual-reporter luciferase assays showed that overexpression of either YAP1 or TEAD1 in HCT116 cells stimulated the promoter activity of SNHG16 (Fig. 6J). And evidently, the promoter activity was most significant when we simultaneously overexpress both of YAP1 and TEAD1 (Fig. 6J). To verify the binding of SNHG16 and TEAD1, we generated a series of 5' deletion constructs of SNHG16 promoter. According to the result of luciferase assay, the regulatory region between - 1794 and -1367 was responsible for YAP1/TEAD1-mediated promoter regulation (Fig. 6K). As shown in Fig. 6l, two binding sites are located in this region. Compared with wild-type (WT), YAP1/TEAD1 failed to stimulate the mutants of both predicted sites in the promoter region of SNHG16 in HCT116 (Fig. 6L). Furthermore, in the ChIP assay, we designed two primer sets containing site 1 and site 2, respectively. Then, we used the primer and purified DNA among ChIP to amplify part of the promoter region. ChIP assays revealed that TEAD1 directly bound to both site1 and site2 of SNHG16 promoter in HCT116 (Fig. 6M). 
The results indicated that YAP1 could directly bind with TEAD1 to form a complex, which could, in turn, bind to the promoter of SNHG16 and regulate its transcription. During this process, YAP1 is indispensable to TEAD1 and these two act synergistically.

\section{The effect of YAP1-SNHG16 positive feedback loop on the progression of tumor}

We have demonstrated that SNHG16 was a direct target of YAP1/TEAD1 complex, and there existed a positive feedback regulation between SNHG16 and YAP1. As aforementioned, YAP1 could regulate the expression of SNHG16 and promote tumor progression. To investigate the YAP1/TEAD1-SNHG16 positive feedback loop on tumor progression, the following experiment was performed. Clone formation revealed that the overexpression of YAP1 significantly promoted the tumor proliferation, whereas the enhanced proliferation of HCT116 was impaired by simultaneous knockdown of SNHG16 (Fig S4A). Similarly, SNHG16 knockdown could partially attenuate the enhanced migration, invasion, and EMT of Lv-Oe-YAP1 stable cell line (Fig S4C, Fig S4E, and Fig. 6Q). In addition, the overexpression of SNHG16 could rescue the inhibitory effect of Lv-anti-YAP1 on tumor progression (Fig S4B, Fig S4D, Fig S4F and Fig. $6 \mathrm{~N}$ ).

\section{The alteration of IncRNA-SNHG16 expression influenced the CRC tumorigenesis and ${ }^{\mathrm{M}} \mathrm{CTC}$ generation in vivo}

To verify the above results in vivo, nude mouse xenograft experiment was performed. The stable cell lines of Lv-anti-SNHG16-NC (HCT116), Lv-anti-SNHG16 (HCT116), and Lv-anti-SNHG16/Lv-Oe-YAP1 (HCT116) were injected into the flanks of nude mice separately. During the period of tumor growth, both the tumor weight and tumor volume was measured. Evidently, knockdown of SNHG16 inhibited the growth of tumor. Furthermore, the inhibitory effect of SNHG16 on tumor growth was rescued by YAP1 overexpression (Fig. 7A, B and C).

Similarly, Ki67 staining also decreased in Lv-anti-SNHG16 group, indicating the inhibited proliferation of tumor. Meanwhile, overexpression of YAP1 significantly rescued the inhibited expression of Ki67 (Fig. 7D). Significantly, the IHC staining also demonstrated the in vitro result that SNHG16 could regulate the expression of YAP1 and the process of EMT. Moreover, YAP1 overexpression could rescue SNHG16-knockdown-mediated inhibitory effect on EMT (Fig. 7D). Through the process of EMT, cancer cells could shed from tumor and invade into blood, thereby forming CTCs and facilitating tumor metastasis. Thus, we detected the CTC from mouse blood (Fig. 7E). Obviously, Lv-anti-SNHG16 significantly decreased the generation ratio of ${ }^{\mathrm{M}} \mathrm{CTC}$, compared with other groups (Fig. 7F); meanwhile, YAP1 overexpression could rescue SNHG16-knockdown-mediated inhibitory effect on ${ }^{\mathrm{M}} \mathrm{CTC}$ generation (Fig. 7F).

The stable cell lines of Lv-anti-SNHG16 NC (HCT116), Lv-anti-SNHG16 (HCT116), Lv-anti-SNHG16/Lv-Oe-YAP1 (HCT116) were separately injected into tail vein to confirm the effect of SNHG16 on tumor metastasis. The representative images of CRC liver metastasis and lung metastasis were presented in Fig. 7G and 7H, respectively. Further analysis revealed that the knockdown of SNHG16 significantly inhibited the metastasis of tumor. In addition, the overexpression of YAP1 could rescue the inhibitory effect of SNHG16 knockdown on tumor metastasis (Fig. 7G and 7H). Collectively, these results demonstrated that SNHG16 could promote the tumor growth, metastasis, and ${ }^{\mathrm{M}} \mathrm{CTC}$ generation. And what's more, YAP1 could rescue the effect of SNHG16 on tumor metastasis.

We summarized our mechanistic findings in a schematic (Fig. 7l). Our study illustrated a positive feedback loop between IncRNA SNHG16 and YAP1/TEAD1. Mechanistically, SNHG16 could act as miRNA sponge to sequester miR-195-5p on Ago2, thereby protecting YAP1 from repression and facilitating CRC liver metastasis. Moreover, YAP1 could also combine with TEAD1 to form a YAP1/TEAD1 complex, which could bind with the promoter of SNHG16 to regulate its transcription. In a positive feedback loop, SNHG16 promoted the migration, invasion, ${ }^{\mathrm{M}} \mathrm{CTC}$ generation, lung metastasis, and liver metastasis of CRC.

\section{Discussion}

Extensive efforts in past have been made to explore the cellular and molecular mechanism of CRC liver metastasis, one of the main cause for the failure of advanced CRC. Recently, IncRNAs have gained enormous attention as a critical regulator of tumor invasion and metastasis ${ }^{57,58}$. Tang et al. also found that, Rho/ROCK signaling-associated IncRNAs is useful for cancer metastasis ${ }^{59}$. In present study, we discovered a positive feedback loop of SNHG16-YAP1/TEAD1 in CRC. Through this positive feedback loop, SNHG16 could

Page $13 / 27$ 
promote $\mathrm{CRC}$ cellular proliferation, migration, invasion, EMT, ${ }^{\mathrm{M}} \mathrm{CTC}$ generation, and liver metastasis of $\mathrm{CRC}$. In turn, YAP1 could combine with TEAD1 to form a complex, which in turn bind with the promoter of SNHG16 and activates its transcription. Therefore, our research clarified a hitherto unexplored mechanism for both SNHG16 and YAP1/TEAD1 in CRC progression.

Clinically, elevated level of IncRNA-SNHG16 located in CRC tissues was positively correlated with TNM stage, LVI, PNI, distant metastasis, and poor prognosis of cancer patients, indicating their potential regulation on CRC dissemination and invasion. Recently, accumulating evidences revealed that IncRNA was associated with the EMT of tumor cells ${ }^{60,61}$. In this study, our results potently demonstrated that SNHG16 exerted oncogenic role in CRC through promoting cell proliferation, mobility, and EMT. CTCs, which was considered as the precursors of metastases ${ }^{13}$. According to recent research, CTCs shed from primary tumor could gain more mesenchymal traits by EMT program, which allow them to have stronger invasive ability and metastatic ability ${ }^{62}$. Qi et al. also demonstrated that, ${ }^{\mathrm{M}} \mathrm{CTC}$ percentage $\geq 2 \%$ prior to resection were significantly associated with early recurrence, multi-intrahepatic recurrence, and lung metastasis in $\mathrm{HCC}^{63}$. Currently, our result revealed that ${ }^{\mathrm{M}} \mathrm{CTC}$ ratio was significantly downregulated after SNHG16 knockdown. Furthermore, we found SNHG16 knockdown significantly inhibited the liver metastasis and lung metastasis of CRC. Given the key role of transcription factors in the process of EMT activation, we screen a panel of EMT-related transcription factors. Among them, YAP1 was the most significant changed in the process of loss- and gain-of-function for SNHG16. According to previous research, YAP1 plays vital roles in the proliferation, invasion, EMT, and metastasis of many cancers ${ }^{64-67}$. In addition, YAP1 is also implicated in the EMT of mammary epithelial cells ${ }^{68,69}$. In our recent research, we also demonstrated the role of miR-195-5p/ Hippo-YAP in EMT activation $^{43}$. Thus, we hypothesized that SNHG16 could affect the process of EMT through YAP1. Consistent with previous studies ${ }^{70,71}$, our study potently demonstrated that YAP1 could significantly promote cell proliferation, migration, invasion, and EMT in CRC. Next, we also found an positive correlation between SNHG16 expression and YAP1 expression in CRC tissue, further proved SNHG16 could regulate YAP1 expression. Subsequently, function analysis revealed that YAP1 could rescue SNHG16-mediated-effect on CRC metastasis in vitro and in vivo. To our knowledge, this study provides the first evidence that SNHG16 could regulate the process of EMT through YAP1, thus facilitating the ${ }^{\mathrm{M}}$ CTC generation and Liver metastasis of CRC.

Currently, mounting evidence suggested that IncRNAs could act as sponges of miRNAs to impact the expression of mRNA ${ }^{49}$. For instance, IncRNA-ATB could act as miRNA sponge to inhibit miR-200, thus regulate the expression of ZEB1 and induce epithelialmesenchymal transition ${ }^{72}$. In addition, IncRNA-HOXD-AS1, which is highly expressed in HCC, could act as a 'sponge' to prevent SOX4 from miR-130-3p-mediated degradation, leading to the HCC metastasis ${ }^{58}$. Subsequent ISH experiment in our research showed that SNHG16 is mainly located in cytoplasm in CRC, which indicating a potential role in posttranscriptional regulation. Thus, we speculated that SNHG16 could act as microRNA sponge to prevent YAP1 from the microRNA mediated inhibition. Here, the inverse correlation between SNHG16 expression and miR-195-5p further indicated the potential targeted regulation between SNHG16 and miR-195-5p. Using bioinformatic analyses, biotin-labeled miR-195-5p pull down assay, anti-Ago2 RIP assay, and luciferase assay, we strongly demonstrated that SNHG16 could directly binds and negatively regulates miR-195-5p expression. Given that both IncRNA SNHG16 and YAP1 interact with miR-195-5p, indicating that SNHG16 may regulate YAP1 expression by competing miR-195-5p. Unsurprisingly, our research revealed that miR-195-5p could rescue the effect of SNHG16 on 3'-UTR luciferase activity of YAP1, expression level of YAP1, and tumor progression. For the first time, we strongly confirmed that SNHG16 could act as miRNA sponge to sequester miR-195-5p, thereby protecting YAP1 from repression and facilitating tumor progression. Interestingly, Chen et al and He et al also reported that IncRNA-SNHG16 could promote CRC progression through miR-124-3p and miR-132-3p $p^{73,74}$, respectively. Different from this two research, we also deeply elucidated the upstream and downstream of SNHG16. And, what's more, we further revealed a positive feedback loop between SNHG16 and YAP1/TEAD1 complex, which could facilitate ${ }^{\mathrm{M}} \mathrm{CTC}$ generation and CRC liver metastasis. However, it remains unclear which microRNA affects most amongst these three microRNAs in CRC. Besides, considering the complex function of IncRNA in cancer $25,26,75$, whether SNHG16 could bind certain proteins or regulate other pathway to exert his effect on CRC progression remains mysterious. These issues is planned to be further investigated in recent research. Collectively, these findings indicate that, the crosstalk between RNAs, e.g., IncRNA and mRNA, forms a large-scale regulatory network among different pathway. Thus, ceRNA networks may pave a new way for tumor therapeutic regimens.

The positive regulation of SNHG16 on YAP1 drew us to investigate the role of YAP1 on SNHG16 expression. Evidently, we demonstrated that YAP1 could activate the expression of SNHG16 for the first time. However, the molecular mechanism underlying YAP1-IncRNA interaction remains unknown. According to previous research, YAP1 cannot regulate transcription alone but combine with downstream transcription factors $54,56,76$, which mainly consist of TEAD1-477. Through N-terminal TEA DNA binding domain, TEAD could bind to the $\mathrm{C}$-terminal region of YAP $1^{78,79}$. Once bound to TEAD, YAP1 relies on the DNA binding domain of TEAD to initiate downstream gene

Page $14 / 27$ 
transcription ${ }^{54,56,76}$. For insistence, Shen et al. found that YAP1/TEAD1 complex could transform NFs to CAFs by regulating SRC ${ }^{80}$. Lamar also found that, YAP could promote metastasis through its TEAD-interaction domain ${ }^{69}$. Currently, we found the potential downstream transcription factors TEAD1 via UCSC, JASPAR, and TRANSFAC. By performing Co-IP and GST-pull down, we demonstrated the direct interaction between YAP1 and TEAD1. We then revealed that, the knockdown of either YAP1 or TEAD1 could inhibit the expression of SNHG16, and the inhibitory effect was most significant when we knocked down both TEAD1 and YAP1 simultaneously. Further research demonstrated that, only when YAP1 and TEAD1 act synergistically can they regulate the transcription of SNHG16. Unsurprisingly, dual-luciferase assay and ChIP assay showed that TEAD1 could bind to the predicted sites of SNHG16 promoter. Herein, we potently demonstrated that YAP1 could directly combine with TEAD1, forming a complex; which could bind to the promoter of SNHG16 and regulate its transcription. Finally, we revealed that YAP1/TEAD1-SNHG16 loop significantly promoted the tumor progression, and SNHG16 could rescue the effect of YAP1 on tumor progression. Therefore, SNHG16 and YAP1 formed a positive feedback regulation on tumor progression.

\section{Conclusions}

Overall, we demonstrated that SNHG16 was upregulated in CRC and significantly associated with poor prognosis of CRC patients. According to Cox-regression analysis, SNHG16 may represent an independent prognostic biomarker in CRC patients. Loss- and gain-of function analysis revealed that, SNHG16 could regulate CRC proliferation, migration, invasion, EMT, ${ }^{\mathrm{M}} \mathrm{CTC}$ generation, and liver metastasis. Next, we demonstrated that YAP1 was the function mediator of SNHG16, and SNHG16 fulfil its oncogenic role in an YAP1 dependent-manner. Mechanistically, SNHG16 could act as miRNA sponge to sequester miR-195-5p on Ago2, thereby protecting YAP1 from repression and facilitating tumor progression. Moreover, YAP1 could also combine with TEAD1 to form a YAP1/TEAD1 complex, which could in turn bind with the promoter of SNHG16 to regulate its transcription. Finally, we revealed that YAP1/TEAD1-SNHG16 loop significantly promoted the tumor progression, and SNHG16 could rescue the effect of YAP1 on tumor progression. Thus, there exists a positive feedback loop between YAP1/TEAD1 and SNHG16. These novel results suggested that SHNG16-YAP1 positive feedback loop may be a potential therapeutic target for CRC.

\section{Abbreviations}

3'-UTR: 3'-untranslated region; CRC: Colorectal cancer; CTC: circulating tumor cell; ${ }^{\mathrm{E}} \mathrm{CTC}$ : epithelial circulating tumor cell; ${ }^{\mathrm{M}} \mathrm{CTC:}$ mesenchymal circulating tumor cell; EMT: Epithelial-mesenchymal transition; LncRNA-SNHG16: Long noncoding RNA SNHG16; miR: MicroRNA/miRNA; miR-195-5p: Hsa-microRNA-195-5p; NC: Negative control; PANT: Paired adjacent normal tissue; qRT-PCR: Quantitative reverse transcription polymerase chain reaction; SD: Standard deviation; YAP1: Yes-associated protein 1; LV-Oe-SNHG16: lentivirus mediated overexpression of SNHG16; Lv-Oe-SNHG16 NC: nonspecific Lentiviral vectors of SNHG16 overexpression; Lv-OeYAP1: lentivirus mediated overexpression of YAP1; LV-Oe-YAP1 NC: nonspecific Lentiviral vectors of YAP1 overexpression; LVI: lymphovascular invasion; PNI perineural invasion; TI: tumor invasion; LNM: lymph node metastasis; TNM: tumor-node-metastasis; CA19-9: carbohydrate antigen 19-9; CEA: carcinoembryonic antigen. IHC: immunohistochemistry.

\section{Declarations}

Acknowledgements

The authors appreciate Wuhan YZY Medical Science and Technology Co., Ltd. (Wuhan, China) for providing equipment and excellent technical support for CTC isolation and identification. We also thank Sze Ka Lun for his helpful language revisions.

Authors' contribution

Participated in research design: Zhenxian Xiang, Bin xiong, Shuyi Wang.

Performed data analysis: Zhenxian Xiang, Bin xiong, Sihao Huang.

Experimental operation: Zhenxian Xiang, Haitao Wu, Guoquan Huang, Qiuming He, Rongzhang Dou.

Wrote or contributed to the writing of the manuscript: Zhenxian Xiang, Qing Liu, Yan Fang, Jialing Song.

Funding

Page $15 / 27$ 
This study was supported by grants from National Natural Science Foundation of China (81872376), National Natural Science Fund Youth Fund of China (81702411), and Health Commission of Hubei Province Scientific Research Project (WJ2019H012).

Availability of data and materials

The data supporting our findings can be found in the supplementary data.

Competing interest

The authors declare no conflict of interest

CONsent for publication

Not applicable

Ethics approval and consent to participate

The research protocol was reviewed and approved by the Ethics Committee of Wuhan University, and informed consent was obtained from all participated patients.

\section{References}

1. Siegel RL, Miller KD, Jemal A. Cancer statistics. 2016. CA: a cancer journal for clinicians. 2016;66(1):7-30.

2. Xu L, Li X, Cai M, et al. Increased expression of Solute carrier family 12 member 5 via gene amplification contributes to tumour progression and metastasis and associates with poor survival in colorectal cancer. Gut. 2016;65(4):635-46.

3. Miller KD, Nogueira L, Mariotto AB, et al. Cancer treatment and survivorship statistics, 2019. CA: a cancer journal for clinicians. 2019;69(5):363-85.

4. Torre LA, Bray F, Siegel RL, Ferlay J, Lortet-Tieulent J, Jemal A. Global cancer statistics, 2012. CA: a cancer journal for clinicians. 2015;65(2):87-108.

5. van de Velde CJ, Boelens PG, Borras JM, et al. EURECCA colorectal: multidisciplinary management: European consensus conference colon \& rectum. Eur J Cancer. 2014;50(1):1 e1-1 e34.

6. Mantke R, Schmidt U, Wolff S, Kube R, Lippert H. Incidence of synchronous liver metastases in patients with colorectal cancer in relationship to clinico-pathologic characteristics. Results of a German prospective multicentre observational study. Eur J Surg Oncol. 2012;38(3):259-65.

7. Quail DF, Joyce JA. Microenvironmental regulation of tumor progression and metastasis. Nat Med. 2013;19(11):1423-37.

8. Lopez-Soto A, Gonzalez S, Smyth MJ, Galluzzi L. Control of Metastasis by NK Cells. Cancer Cell. 2017;32(2):135-54.

9. Pantel K, Brakenhoff RH. Dissecting the metastatic cascade. Nat Rev Cancer. 2004;4(6):448-56.

10. Mani SA, Guo W, Liao MJ, et al. The epithelial-mesenchymal transition generates cells with properties of stem cells. Cell. 2008;133(4):704-15.

11. Yang J, Mani SA, Donaher JL, et al. Twist, a master regulator of morphogenesis, plays an essential role in tumor metastasis. Cell. 2004;117(7):927-39.

12. Yang J, Weinberg RA. Epithelial-mesenchymal transition: at the crossroads of development and tumor metastasis. Dev Cell. 2008;14(6):818-29.

13. Au SH, Storey BD, Moore JC, et al. Clusters of circulating tumor cells traverse capillary-sized vessels. Proc Natl Acad Sci U S A. 2016;113(18):4947-52.

14. Thiery JP. Epithelial-mesenchymal transitions in tumour progression. Nat Rev Cancer. 2002;2(6):442-54.

15. Rhim AD, Mirek ET, Aiello NM, et al. EMT and dissemination precede pancreatic tumor formation. Cell. 2012;148(1-2):349-61.

16. Joosse SA, Gorges TM, Pantel K. Biology, detection, and clinical implications of circulating tumor cells. EMBO molecular medicine. 2015;7(1):1-11.

17. Lohr JG, Adalsteinsson VA, Cibulskis K, et al. Whole-exome sequencing of circulating tumor cells provides a window into metastatic prostate cancer. Nature biotechnology. 2014;32(5):479-84. 
18. Mohamadi RM, Besant JD, Mepham A, et al. Nanoparticle-mediated binning and profiling of heterogeneous circulating tumor cell subpopulations. Angew Chem Int Ed Engl. 2015;54(1):139-43.

19. Fan Z, Shelton M, Singh AK, Senapati D, Khan SA, Ray PC. Multifunctional plasmonic shell-magnetic core nanoparticles for targeted diagnostics, isolation, and photothermal destruction of tumor cells. Acs Nano. 2012;6(2):1065-73.

20. Ferreira MM, Ramani VC, Jeffrey SS. Circulating tumor cell technologies. Molecular oncology. 2016;10(3):374-94.

21. Kallergi G, Papadaki MA, Politaki E, Mavroudis D, Georgoulias V, Agelaki S. Epithelial to mesenchymal transition markers expressed in circulating tumour cells of early and metastatic breast cancer patients. Breast Cancer Res. 2011;13(3):R59.

22. Habli Z, AlChamaa W, Saab R, Kadara H, Khraiche ML. Circulating Tumor Cell Detection Technologies and Clinical Utility: Challenges and Opportunities. Cancers (Basel). 2020;12(7).

23. Dasgupta A, Lim AR, Ghajar CM. Circulating and disseminated tumor cells: harbingers or initiators of metastasis? Molecular oncology. 2017;11(1):40-61.

24. Jiang Y, Cao W, Wu K, et al. LncRNA LINC00460 promotes EMT in head and neck squamous cell carcinoma by facilitating peroxiredoxin-1 into the nucleus. J Exp Clin Cancer Res. 2019;38(1):365.

25. Wang KC, Chang HY. Molecular mechanisms of long noncoding RNAs. Mol Cell. 2011;43(6):904-14.

26. Chan JJ, Tay Y. Noncoding RNA:RNA Regulatory Networks in Cancer. Int J Mol Sci. 2018;19(5).

27. Huarte M. The emerging role of IncRNAs in cancer. Nat Med. 2015;21(11):1253-61.

28. Mitra R, Chen X, Greenawalt EJ, et al. Decoding critical long non-coding RNA in ovarian cancer epithelial-to-mesenchymal transition. Nat Commun. 2017;8(1):1604.

29. Yu M, Ohira M, Li Y, et al. High expression of ncRAN, a novel non-coding RNA mapped to chromosome 17q25.1, is associated with poor prognosis in neuroblastoma. International journal of oncology. 2009;34(4):931-8.

30. Han W, Du X, Wang J, Sun L, Li Y. WITHDRAWN: SNHG16 indicates a poor prognosis and affects cell proliferation, migration and invasion in non-small cell lung cancer. Exp Cell Res. 2017.

31. Cao X, Xu J, Yue D. LncRNA-SNHG16 predicts poor prognosis and promotes tumor proliferation through epigenetically silencing p21 in bladder cancer. Cancer gene therapy. 2018;25(1-2):10-7.

32. Christensen LL, True K, Hamilton MP, et al. SNHG16 is regulated by the Wnt pathway in colorectal cancer and affects genes involved in lipid metabolism. Molecular oncology. 2016;10(8):1266-82.

33. Zhao W, Fu H, Zhang S, Sun S, Liu Y. LncRNA SNHG16 drives proliferation, migration, and invasion of hemangioma endothelial cell through modulation of miR-520d-3p/STAT3 axis. Cancer Med. 2018.

34. Abylkassov R, Xie Y. Role of Yes-associated protein in cancer: An update. Oncol Lett. 2016;12(4):2277-82.

35. Albrengues J, Bertero T, Grasset E, et al. Epigenetic switch drives the conversion of fibroblasts into proinvasive cancer-associated fibroblasts. Nat Commun. 2015;6.

36. Collak FK, Demir U, Ozkanli S, Kurum E, Zerk PE. Increased expression of YAP1 in prostate cancer correlates with extraprostatic extension. Cancer Biol Med. 2017;14(4):405-13.

37. Dong A, Gupta A, Pai RK, Tun M, Lowe AW. The human adenocarcinoma-associated gene, AGR2, induces expression of amphiregulin through Hippo pathway co-activator YAP1 activation. The Journal of biological chemistry. 2011;286(20):18301-10.

38. Visser S, Yang X. LATS tumor suppressor: a new governor of cellular homeostasis. Cell Cycle. 2010;9(19):3892-903.

39. Zhang WJ, Jiao HL, Zhang XD, et al. Correlation between the expression of DNMT1, and GSTP1 and APC, and the methylation status of GSTP1 and APC in association with their clinical significance in prostate cancer. Mol Med Rep. 2015;12(1):141-6.

40. Zhao B, Wei X, Li W, et al. Inactivation of YAP oncoprotein by the Hippo pathway is involved in cell contact inhibition and tissue growth control. Gene Dev. 2007;21(21):2747-61.

41. Stein C, Bardet AF, Roma G, et al. YAP1 Exerts Its Transcriptional Control via TEAD-Mediated Activation of Enhancers. PLoS Genet. 2015;11(8):e1005465.

42. Zhang X, Xu J, Jiang T, Liu G, Wang D, Lu Y. MicroRNA-195 suppresses colorectal cancer cells proliferation via targeting FGF2 and regulating Wnt/beta-catenin pathway. Am J Cancer Res. 2016;6(11):2631-40.

43. Sun M, Song HB, Wang SY, et al. Integrated analysis identifies microRNA-195 as a suppressor of Hippo-YAP pathway in colorectal cancer. Journal of hematology \& oncology. 2017;10. 
44. Chen F, Wang S, Fang Y, et al. Feasibility of a novel one-stop ISET device to capture CTCs and its clinical application. Oncotarget. 2017;8(2):3029-41.

45. Banyard J, Bielenberg DR. The role of EMT and MET in cancer dissemination. Connect Tissue Res. 2015;56(5):403-13.

46. Lamouille S, Xu J, Derynck R. Molecular mechanisms of epithelial-mesenchymal transition. Nature reviews Molecular cell biology. 2014;15(3):178-96.

47. Zhou Y, Ren H, Dai B, et al. Hepatocellular carcinoma-derived exosomal miRNA-21 contributes to tumor progression by converting hepatocyte stellate cells to cancer-associated fibroblasts. J Exp Clin Cancer Res. 2018;37(1):324.

48. Zhang R, Shi H, Ren F, et al. MicroRNA-338-3p suppresses ovarian cancer cells growth and metastasis: implication of Wnt/catenin beta and MEK/ERK signaling pathways. J Exp Clin Cancer Res. 2019;38(1):494.

49. Salmena L, Poliseno L, Tay Y, Kats L, Pandolfi PP. A ceRNA hypothesis: the Rosetta Stone of a hidden RNA language? Cell. 2011;146(3):353-8.

50. Huang Y, Xiang B, Liu Y, Wang Y, Kan H. LncRNA CDKN2B-AS1 promotes tumor growth and metastasis of human hepatocellular carcinoma by targeting let-7c-5p/NAP1L1 axis. Cancer Lett. 2018;437:56-66.

51. Li JH, Liu S, Zhou H, Qu LH, Yang JH. starBase v2.0: decoding miRNA-ceRNA, miRNA-ncRNA and protein-RNA interaction networks from large-scale CLIP-Seq data. Nucleic acids research. 2014;42(Database issue):D92-7.

52. Paraskevopoulou MD, Vlachos IS, Karagkouni D, et al. DIANA-LncBase v2: indexing microRNA targets on non-coding transcripts. Nucleic acids research. 2016;44(D1):D231-8.

53. Karginov FV, Hannon GJ. Remodeling of Ago2-mRNA interactions upon cellular stress reflects miRNA complementarity and correlates with altered translation rates. Gene Dev. 2013;27(14):1624-32.

54. Li Z, Zhao B, Wang P, et al. Structural insights into the YAP and TEAD complex. Gene Dev. 2010;24(3):235-40.

55. Zhao Q, Cao J, Wu YC, et al. Circulating miRNAs is a potential marker for gefitinib sensitivity and correlation with EGFR mutational status in human lung cancers. Am J Cancer Res. 2015;5(5):1692-705.

56. Pobbati AV, Hong WJ. Emerging roles of TEAD transcription factors and its coactivators in cancers. Cancer Biol Ther. 2013;14(5):390-8.

57. Tan BS, Yang MC, Singh S, et al. LncRNA NORAD is repressed by the YAP pathway and suppresses lung and breast cancer metastasis by sequestering S100P. Oncogene. 2019;38(28):5612-26.

58. Wang H, Huo X, Yang XR, et al. STAT3-mediated upregulation of IncRNA HOXD-AS1 as a ceRNA facilitates liver cancer metastasis by regulating SOX4. Mol Cancer. 2017;16(1):136.

59. Tang Y, He Y, Zhang P, et al. LncRNAs regulate the cytoskeleton and related Rho/ROCK signaling in cancer metastasis. Mol Cancer. 2018;17(1):77.

60. Grelet S, Link LA, Howley B, et al. A regulated PNUTS mRNA to IncRNA splice switch mediates EMT and tumour progression. Nature cell biology. 2017;19(9):1105-15.

61. Dong H, Hu J, Zou K, et al. Activation of LncRNA TINCR by H3K27 acetylation promotes Trastuzumab resistance and epithelialmesenchymal transition by targeting MicroRNA-125b in breast Cancer. Mol Cancer. 2019;18(1):3.

62. Li YM, Xu SC, Li J, et al. Epithelial-mesenchymal transition markers expressed in circulating tumor cells in hepatocellular carcinoma patients with different stages of disease. Cell Death Dis. 2013;4(10):e831.

63. Qi LN, Xiang BD, Wu FX, et al. Circulating Tumor Cells Undergoing EMT Provide a Metric for Diagnosis and Prognosis of Patients with Hepatocellular Carcinoma. Cancer research. 2018;78(16):4731-44.

64. Sun Z, Ou C, Liu J, et al. YAP1-induced MALAT1 promotes epithelial-mesenchymal transition and angiogenesis by sponging miR126-5p in colorectal cancer. Oncogene. 2019;38(14):2627-44.

65. Li Y, Zhang X, Zheng Q, et al. YAP1 Inhibition in HUVECs Is Associated with Released Exosomes and Increased Hepatocarcinoma Invasion and Metastasis. Molecular therapy Nucleic acids. 2020;21:86-97.

66. Huang Z, Su B, Liu F, et al. YAP1 Promotes Tumor Invasion and Metastasis in Nasopharyngeal Carcinoma with Hepatitis B Virus Infection. Onco Targets Ther. 2020;13:5629-42.

67. Li W, Cao Y, Xu J, et al. YAP transcriptionally regulates COX-2 expression and GCCSysm-4 (G-4), a dual YAP/COX-2 inhibitor, overcomes drug resistance in colorectal cancer. J Exp Clin Cancer Res. 2017;36(1):144. 
68. Overholtzer M, Zhang J, Smolen GA, et al. Transforming properties of YAP, a candidate oncogene on the chromosome $11 \mathrm{q} 22$ amplicon. Proc Natl Acad Sci U S A. 2006;103(33):12405-10.

69. Lamar JM, Stern P, Liu H, Schindler JW, Jiang ZG, Hynes RO. The Hippo pathway target, YAP, promotes metastasis through its TEAD-interaction domain. Proc Natl Acad Sci U S A. 2012;109(37):E2441-50.

70. Shao DD, Xue W, Krall EB, et al. KRAS and YAP1 converge to regulate EMT and tumor survival. Cell. 2014;158(1):171-84.

71. Yao P, Li Y, Shen W, et al. ANKHD1 silencing suppresses the proliferation, migration and invasion of CRC cells by inhibiting YAP1induced activation of EMT. Am J Cancer Res. 2018;8(11):2311-24.

72. Yuan JH, Yang F, Wang F, et al. A Long Noncoding RNA Activated by TGF-beta Promotes the Invasion-Metastasis Cascade in Hepatocellular Carcinoma. Cancer Cell. 2014;25(5):666-81.

73. Chen ZY, Wang XY, Yang YM, et al. LncRNA SNHG16 promotes colorectal cancer cell proliferation, migration, and epithelialmesenchymal transition through miR-124-3p/MCP-1. Gene therapy. 2020.

74. He X, Ma J, Zhang M, Cui J, Yang H. Long Non-Coding RNA SNHG16 Activates USP22 Expression to Promote Colorectal Cancer Progression by Sponging miR-132-3p. Onco Targets Ther. 2020;13:4283-94.

75. Jiang H, Li T, Qu Y, et al. Long non-coding RNA SNHG15 interacts with and stabilizes transcription factor Slug and promotes colon cancer progression. Cancer Lett. 2018;425:78-87.

76. Xiao JH, Davidson I, Matthes H, Garnier JM, Chambon P. Cloning, expression, and transcriptional properties of the human enhancer factor TEF-1. Cell. 1991;65(4):551-68.

77. Harvey KF, Zhang X, Thomas DM. The Hippo pathway and human cancer. Nat Rev Cancer. 2013;13(4):246-57.

78. Anbanandam A. Albarado Dc Fau - Nguyen CT, Nguyen Ct Fau - Halder G, Halder G, Fau - Gao X, Gao X, Fau - Veeraraghavan S, Veeraraghavan S. Insights into transcription enhancer factor 1 (TEF-1) activity from the solution structure of the TEA domain. (0027-8424 (Print)).

79. Tian W, Yu J, Fau - Tomchick DR, Tomchick Dr Fau - Pan D, Pan D, Fau - Luo X, Luo X. Structural and functional analysis of the YAP-binding domain of human TEAD2. (1091-6490 (Electronic)).

80. Shen T, Li Y, Zhu S, et al. YAP1 plays a key role of the conversion of normal fibroblasts into cancer-associated fibroblasts that contribute to prostate cancer progression. J Exp Clin Cancer Res. 2020;39(1):36.

\section{Figures}


A

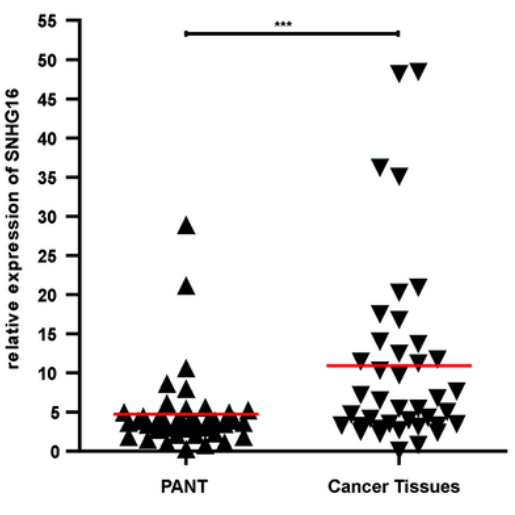

C

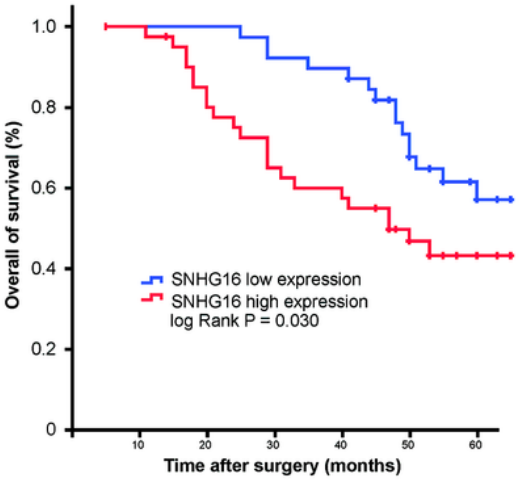

E

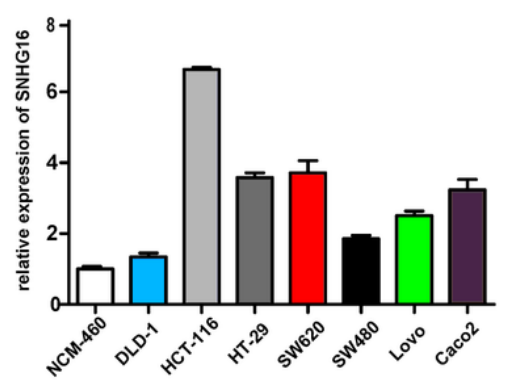

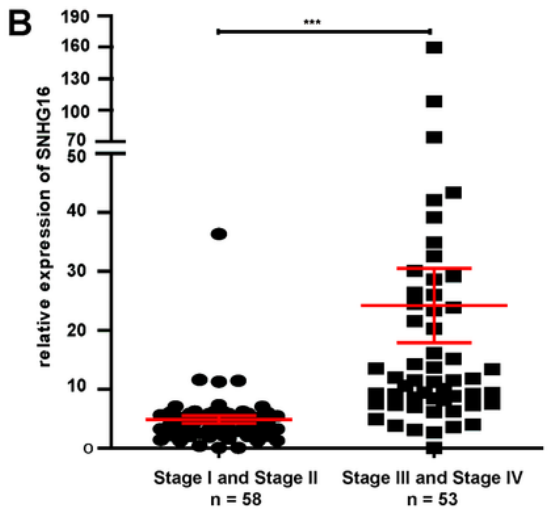

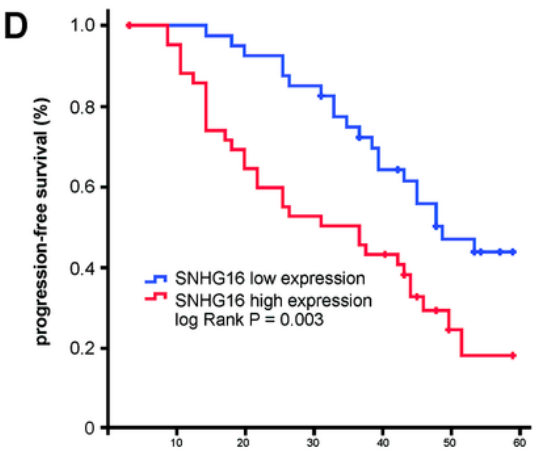

$\mathbf{F}$

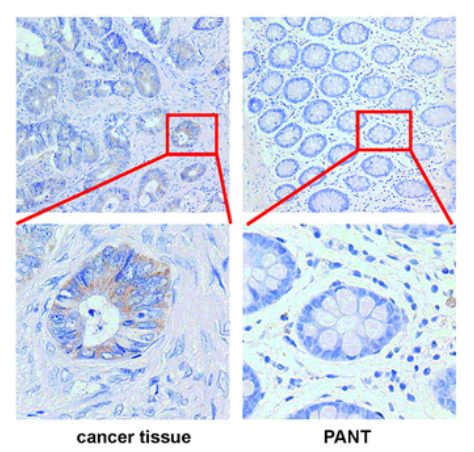

Figure 1

SNHG16 is upregulated in colorectal cancer tissues and indicates poor prognosis of colorectal cancer patients $* \star p<~ 0.01, * \star * p<0.001$. compared with NC group; Data are shown as mean \pm SEM $(n=3)$. A: SNHG16 expression was validated by qRT-PCR in CRC tumor tissue and paired non-tumor tissue; B: Relationship between the expression of SNHG16 and TNM stage of CRC patients. C-D: The relationship between the expression of SNHG16 and overall survival (C) and progression-free survival (D) was analyzed by KaplanMeier survival analysis.. E: The relative expression of SNHG16 in CRC cell lines was validated by PCR. F: Representative images of SNHG16 expression in cancer tissues and adjacent normal tissues were shown by in situ hybridization (ISH) 

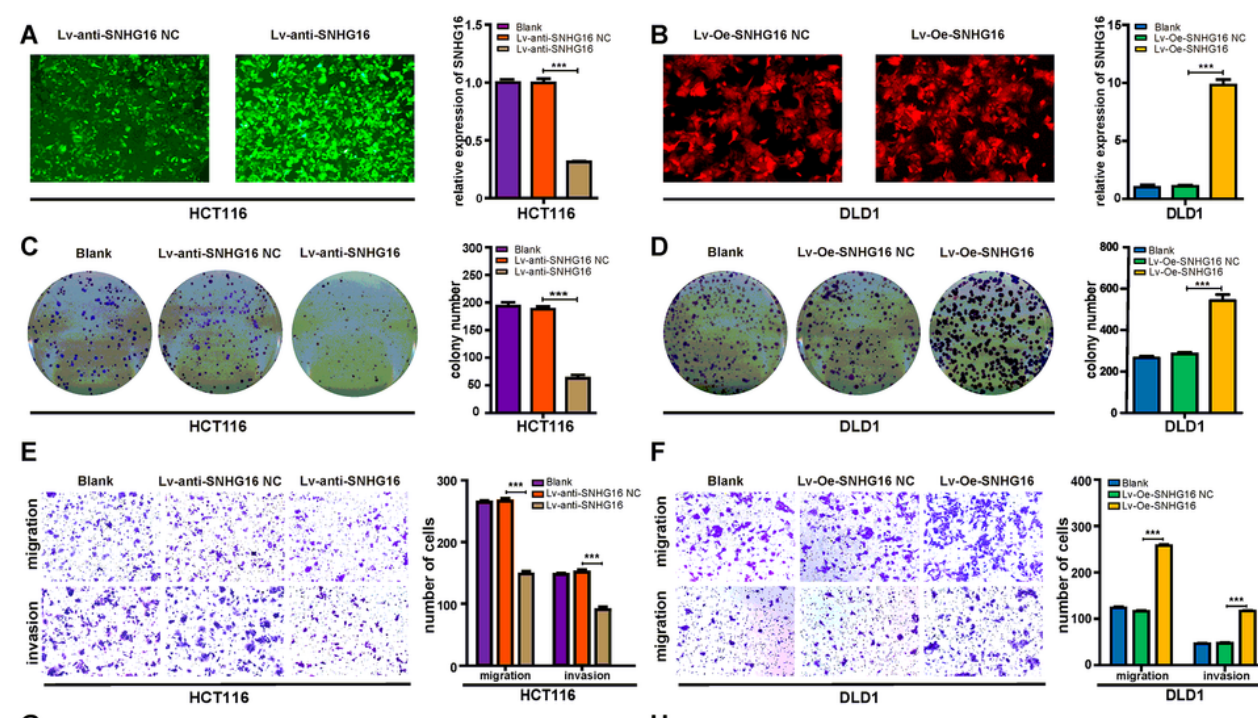

$\mathbf{F}$
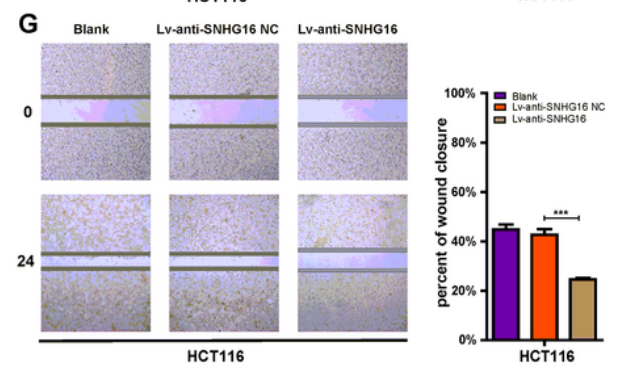

H Blank LV-Oe-SNHG16 NC LV-Oe-SNHG16
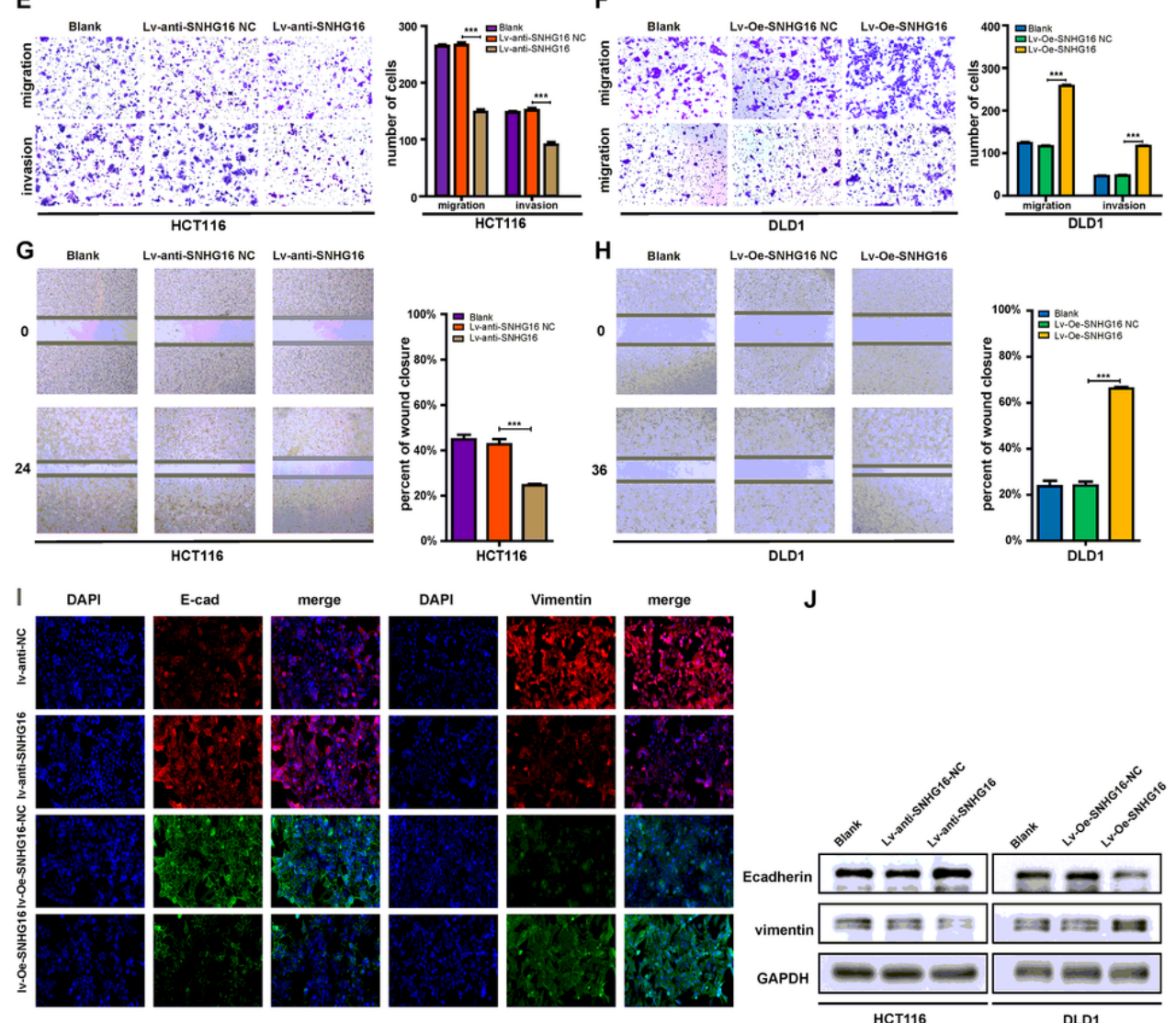

\section{Figure 2}

The ectopic expression of IncRNA-SNHG16 affects proliferation, migration, invasion, and EMT of CRC cells. ${ }^{\star \star} p<0.01,{ }^{* \star *} p<0.001$. compared with NC group; Data are shown as mean \pm SEM $(n=3)$. A: the lentivirus-mediated SNHG16 knockdown in HCT116, and the knockdown effect was validated by qRT-PCR. B: the lentivirus-mediated SNHG16 overexpression in DLD1, and the overexpression effect was validated by qRT-PCR. C-D: colony formation showed that SNHG16 knockdown induced significant decreased colony formation in HCT116 (C), while SNHG16 overexpression increased the colony formation in DLD1 (D). E: Graphical representation of transwell showing decreased migration and invasion of HCT116 after IncRNA-SNHG16 knockdown. F: Graphical representation of transwell showing increased migration and invasion of HCT116 after IncRNA-SNHG16 overexpression. G-H: Graphical representation of wound healing showing decreased migration of HCT116 after IncRNA-SNHG16 knockdown (G), while IncRNA-SNHG16 overexpression significantly increased the migration of DLD1 (H) . I-J: immunofluorescence (I) and western blot $(\mathrm{J})$ showed that SNHG16 could regulate the EMT of CRC cells. 

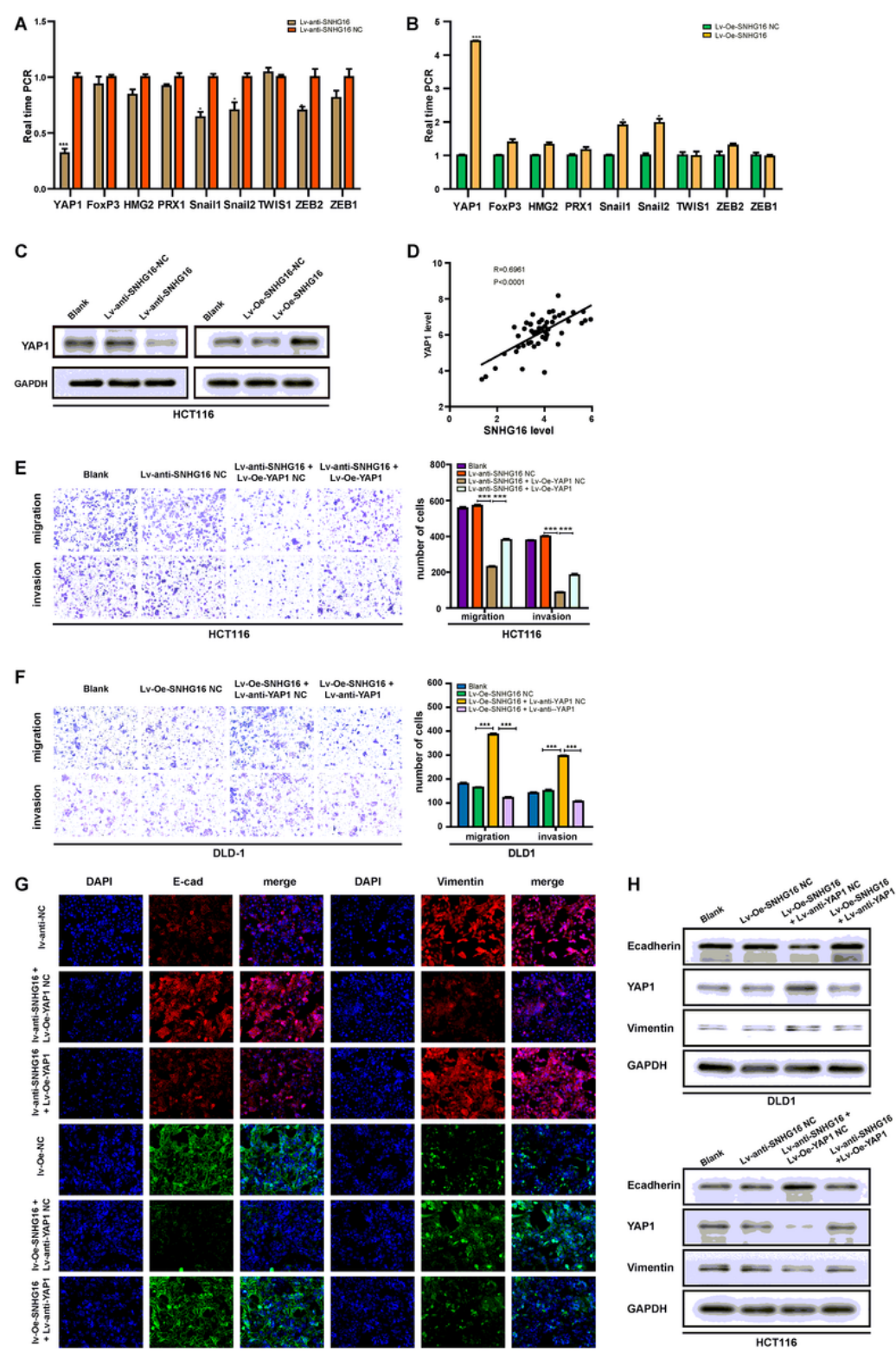

\section{Figure 3}

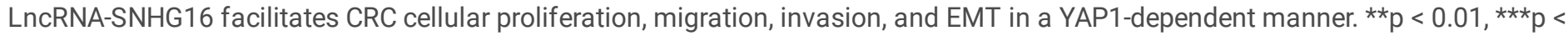
0.001 , compared with NC group. Data are shown as mean \pm SEM $(n=3)$. A-B: Relative expression levels of representative EMT related transcription factors were detected in Lv-anti-SNHG16 (A) or Lv-Oe-SNHG16 (B) transfected HCT116. C: SNHG16 could positively regulate the protein expression of YAP1. D: Pearson correlation analysis revealed an obvious positive association between the expression of SNHG16 and YAP1 in CRC tissues. E: Transwell migration and invasion revealed that SNHG16 knockdown inhibited the migration and invasion of HCT116, and YAP1 overexpression could rescue this effect. F: In addition, YAP1 knockdown could rescue SNHG16-overexpression-mediated effect on migration and invasion. G-H: immunofluorescence $(\mathrm{G})$ and Western blot $(\mathrm{H})$ demonstrated that, YAP1 could rescue the effect of SNHG16 on CRC EMT. 
A
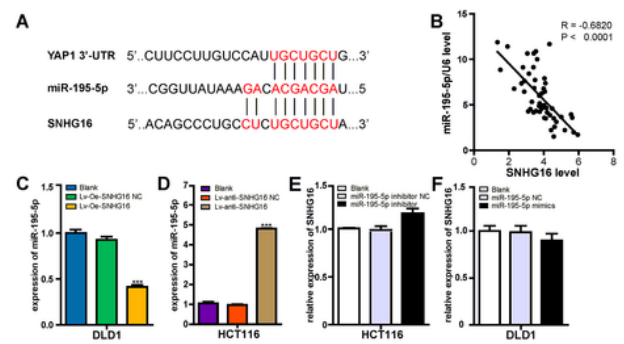

G

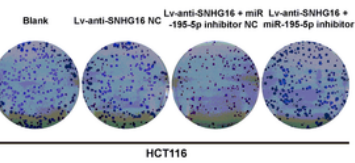

H
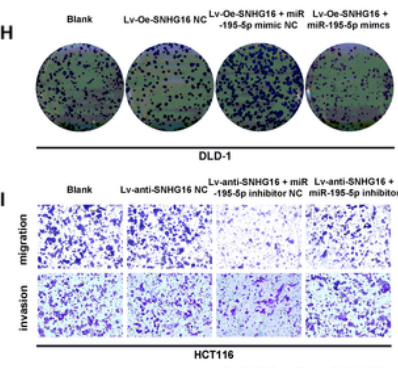

$\mathbf{J}$
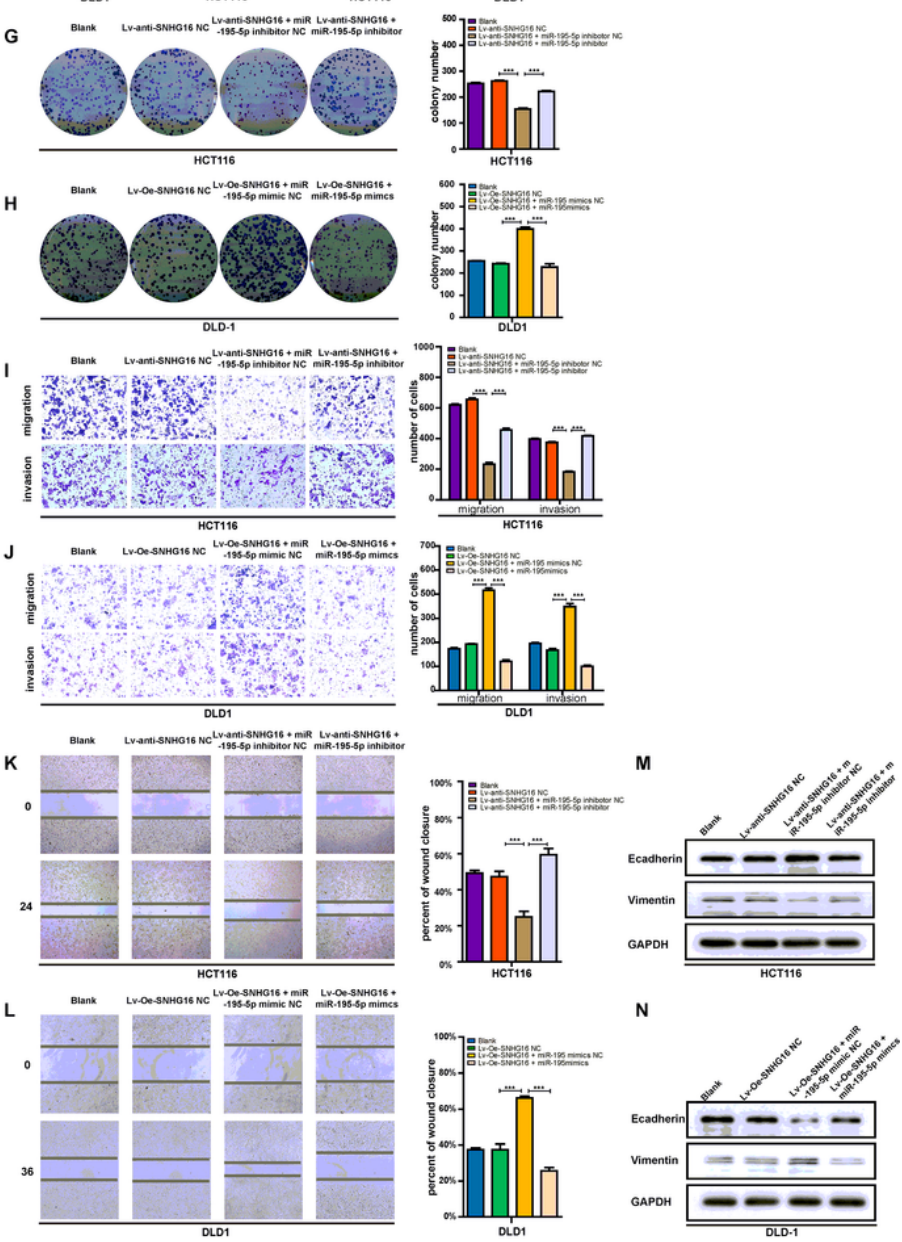

N
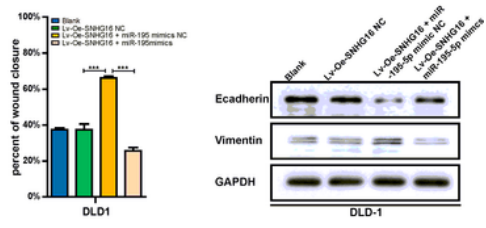

Figure 4

miR-195-5p could potently abrogate the effect of SNHG16/YAP1 axis on tumor progression. **p $<0.01, * \star \star p<0.001$ compared with NC group; Data are shown as mean \pm SEM $(n=3)$ A: The putative miR-195-5p binding sites with the SNHG16 and YAP1 3'-UTR were shown. B: Pearson correlation analysis revealed an obvious negative association between the expression of SNHG16 and miR-195-5p in CRC tissues. C-D: Real-time PCR showed that SNHG16 could negatively regulate miR-195-5p expression in DLD1 (C) and HCT116 (D). E: The relative expression of SNHG16 in miR-195-5p inhibitor or miR-195-5p inhibitor NC transfected HCT116. F :The relative expression of SNHG16 in miR-195-5p mimics or miR-195-5p mimics NC transfected DLD1. G-H: Graphical representation of colony formation shows miR-195-5p inhibitor could rescue the SNHG16-downregulation-mediated inhibitory effect on proliferation (G); meanwhile, miR195-5p mimics could also rescue the effect of SNHG16 overexpression on proliferation (H). I: Transwell assay showed that miR-195-5p inhibitor could largely reverse the inhibitory effects of IncRNA-SNHG16 knockdown on migration and invasion of DLD1. J: Similarly, miR-195-5p mimics could also rescue the promotive effect of IncRNA-SNHG16 overexpression on migration and invasion. K-L: Wound healing assay further demonstrated that miR-195-5p could rescue the effect of IncRNA-SNHG16 on migration. M-N: miR-195-5p inhibitor significantly reversed the inhibited EMT of SNHG16 knockdown (M). Meanwhile, the enhanced EMT of SNHG16 overexpression could be rescued by miR-195-5p mimics $(\mathrm{N})$. 
A

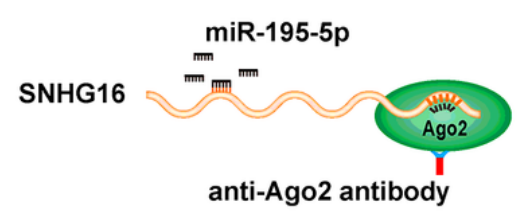

D

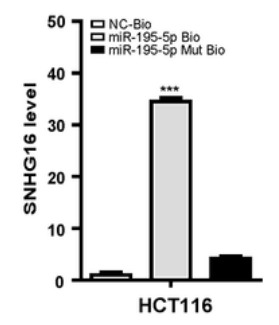

G

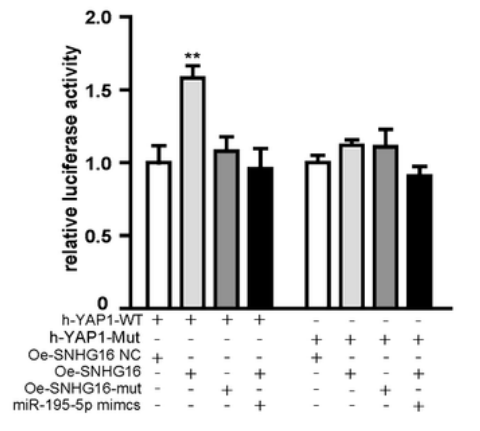

B

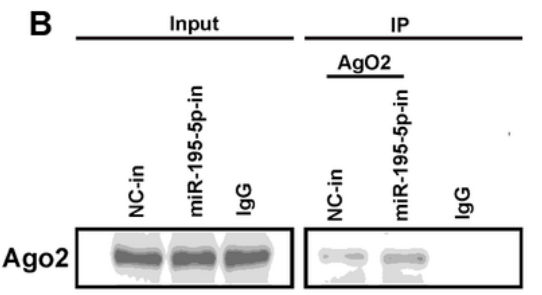

E

SNHG16-Mut: 5'...ACAGCCCUGCCUCACGACGAA... $3^{\prime}$

miR-195-5p: 3'...CGGUUAUAAAGACACGACGAU...5

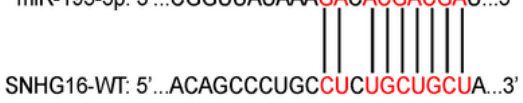

I

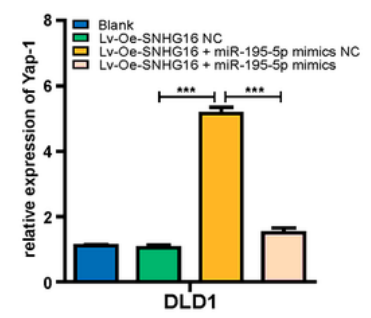

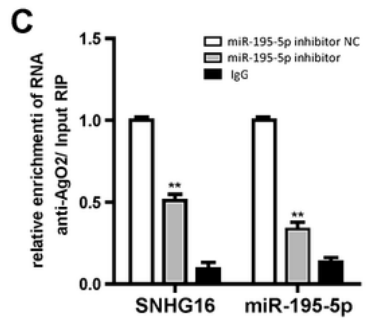

$\mathbf{F}$
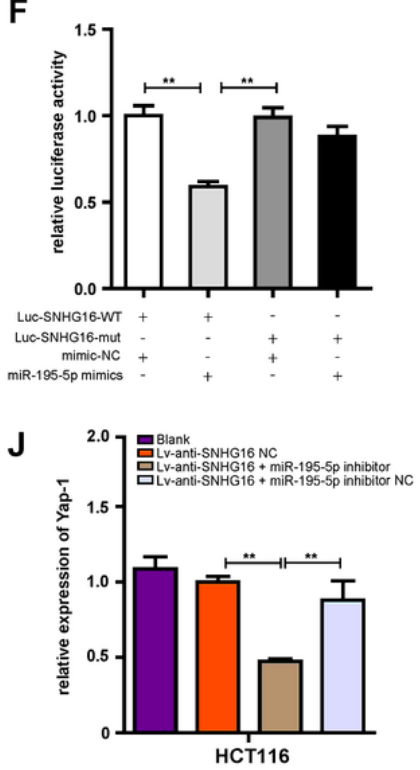

K

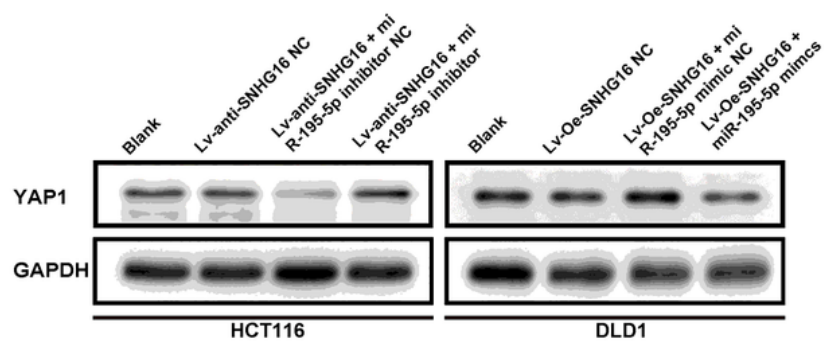

\section{Figure 5}

IncRNA-SNHG16 functions as a ceRNA and sponges miR-195-5p, further regulating YAP1 expression and facilitating tumor progression. ${ }^{* \star} p<0.01,{ }^{* \star} p<0.001$ compared with NC group; Data are shown as mean \pm SEM $(n=3)$ A. schematic illustration of anti-Ago2 RIP strategy, which was performed to validate endogenous miR-195-5p:SNHG16 binding. B. Through Ago2 antibody or IgG antibody, the Ago2 protein was immunoprecipitated from cell extracts and was further detected by western blot. $C$ In the presence of miR-195-5p inhibitor or negative control, the relative expression of SNHG16 and miR-195-5p bound to Ago2 or IgG was measured by RT-qPCR. D: HCT116 was transfected with biotinylated NC (NC-Bio), biotinylated mutant miR-195-5p (miR-195-5p-Mut-Bio) or WT miR-195-5p (miR195-5p-Bio). Cells were collected 36 hours later to perform pulldown assay through qRT-PCR. E: The position of miR-195-5p binding site in SNHG16 is shown. Mutation (underlined) was introduced into SNHG16 to disrupt base-pairing with miR-195-5p seed sequence. F: Dual-luciferase reporter assay was performed to reveal the binding of miR-195-5p to SNHG16.G: Luciferase assay among SNHG16 (OeSNHG16 and Oe-SNHG16 mut), miR-195-5p, and YAP1 (wild and Mut) was performed to confirm the interplay of the three factors.. I-K Through western blot and qRT-PCR, we found SNHG16 could positively regulate the expression of YAP1. Furthermore, miR-195-5p could rescue SNHG16-mediated positive regulation on YAP1. 

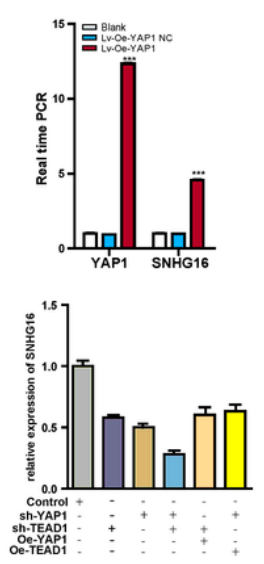

B

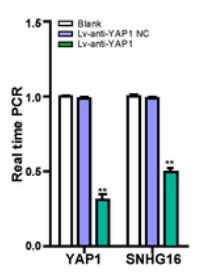

E

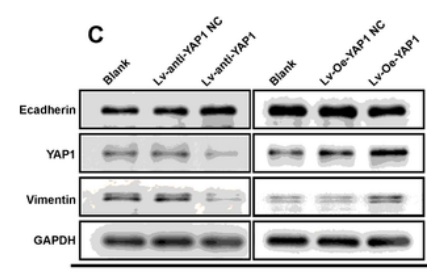

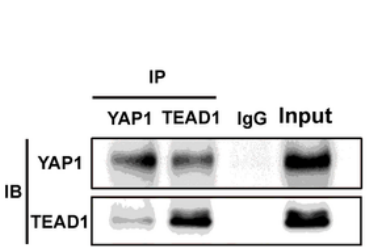
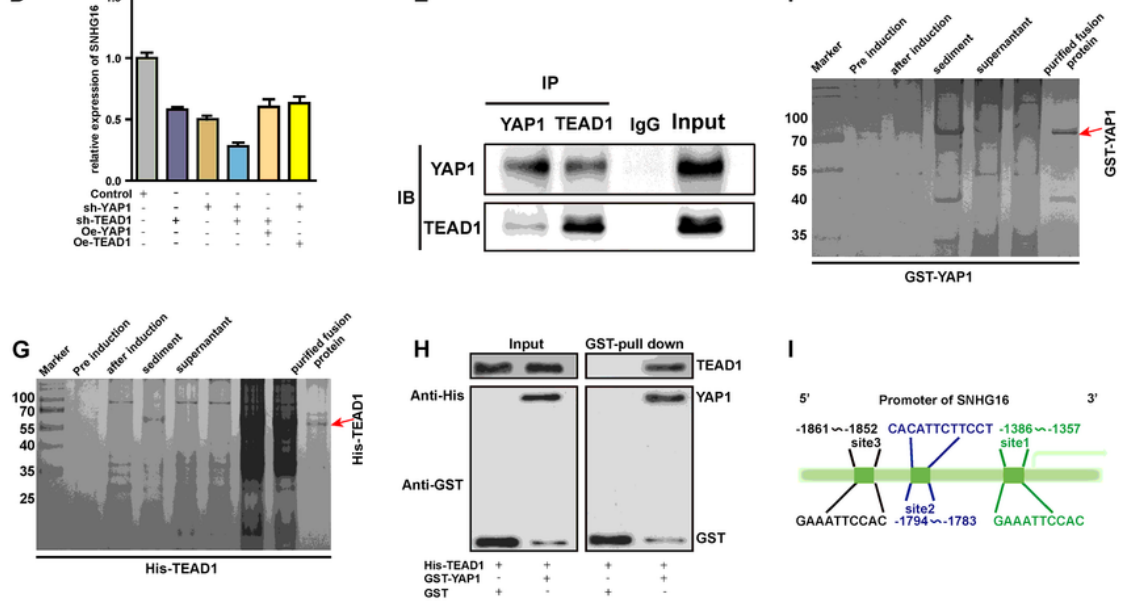

I

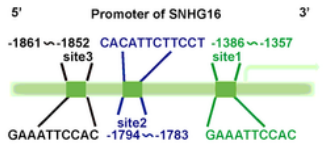

$\mathbf{J}$
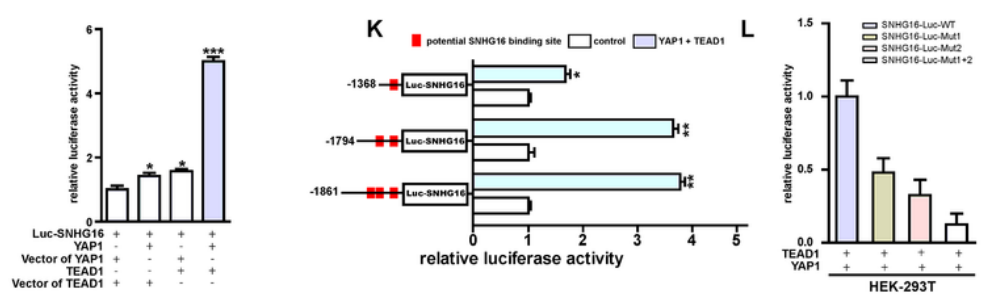

M
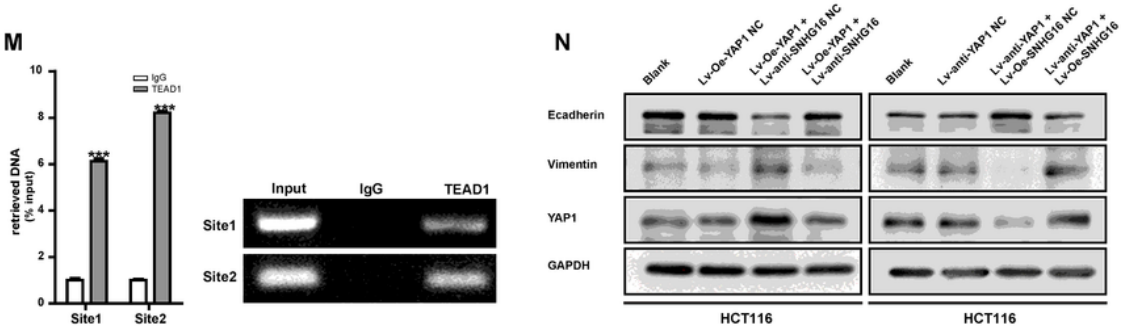

\section{Figure 6}

YAP1 could combine with TEAD1, forming a complex that binds to the promoter region of SNHG16 and activates its transcription. . **p $<0.01$, ${ }^{* \star} p<0.001$ compared with NC group; Data are shown as mean \pm SEM $(n=3)$. A: RT-qPCR results revealed that the overexpression of YAP1 could upregulate the expression of IncRNA-SNHG16 in HCT116. B: Meanwhile, the expression of SNHG16 could also be reduced by knockdown of YAP1. C: western blot showed that YAP1 knockdown inhibited the EMT of CRC, while YAP1 overexpression promoted the EMT of HCT116. D: qRT-PCR was used to detect the expression of SNHG16 when we changed the level of either YAP1 or TEAD1. E: Co-IP experiments showed that YAP1 could bind to TEAD1 directly or indirectly in HCT116. F: The GST-tagged YAP1 was successfully induced and purified from Escherichia coli. G: The His-tagged TEAD1 was also successfully induced and purified from Escherichia coli. H: GST pull down assay further demonstrated the direct interaction between YAP1 and TEAD1. I: Schematic diagram showing the human SNHG16 upstream promoter region (top), including the predicted TEAD1-binding regions. J: Dual-reporter luciferase assays showed that overexpression of YAP1 or TEAD1 in HCT116 cells stimulated the promoter activity of SNHG16, and the activity was most significant when we simultaneously overexpress both of YAP1 and TEAD1. K: Selective mutation analyses identified YAP1/TEAD1-complex-responsive-regions in the SNHG16 promoter in HCT116 cells. L: Compared with wild-type (WT), YAP1/TEAD1 failed to stimulate the mutants of both predicted sites in the promoter regions of SNHG16 in HEK-294T. Mヌ ChIP assay demonstrated the direct binding of TEAD1 to the SNHG16 promoter, including CHIP1 and CHIP2 in HCT116 cells. Input, 10\% of total lysate. N: Evidently, SNHG16 knockdown could partially attenuated the enhanced EMT of YAP1 overexpression. In addition, the overexpression of SNHG16 could rescue the inhibitory effect of YAP1 knockdown on tumor EMT. 

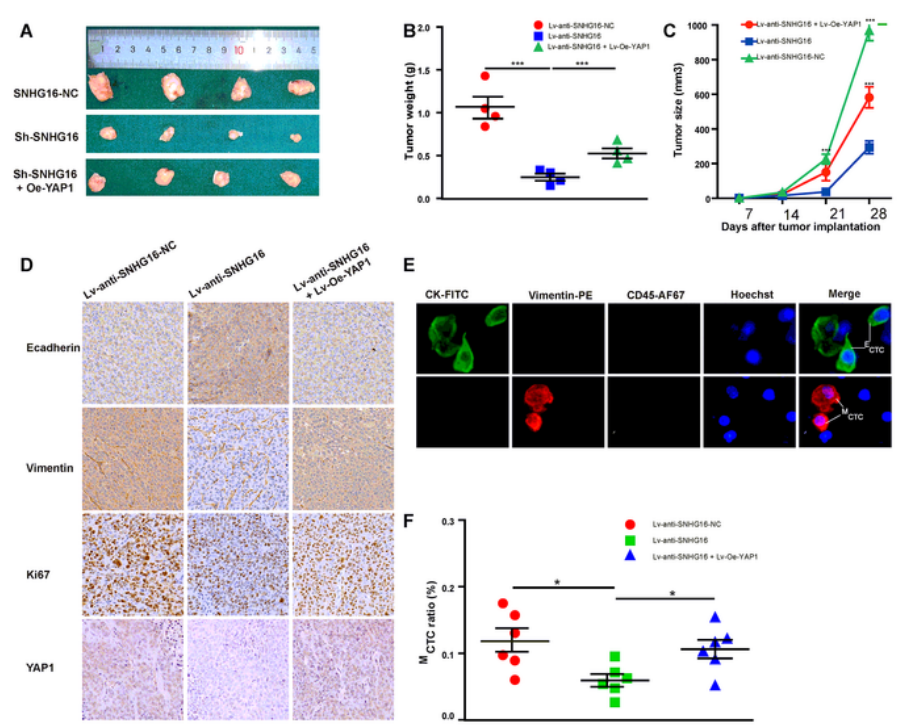

E
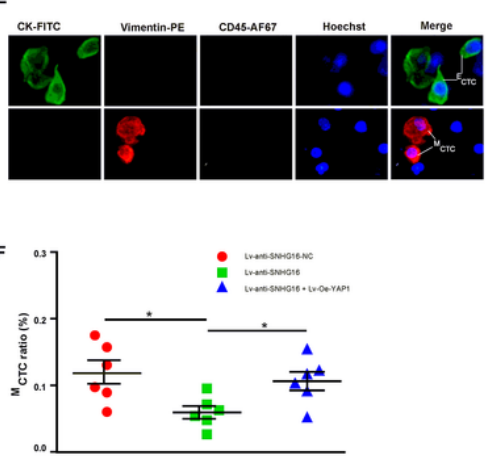

G

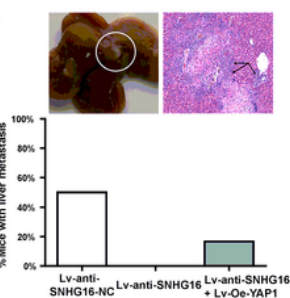

H

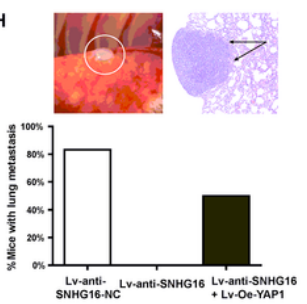

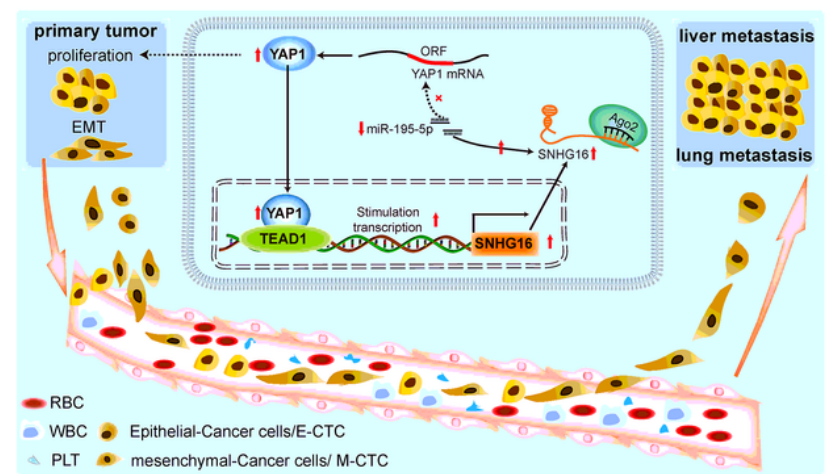

\section{Figure 7}

The alteration of SNHG16 expression influenced the CRC tumorigenesis and CTC generation in vivo. $\star \star p p<0.01$, $\star \star \star p ~<~ 0.001$. compared with NC group; Data are shown as mean \pm SEM $(n=3)$. A-C: The morphological characteristics $(A)$, weight $(B)$, and size $(C)$ of tumor xenograft in the Lv-anti-SNHG16 NC group, Lv-anti-SNHG16 group, and Lv-anti-SNHG16 + Lv-Oe-YAP1 groups. Error bars, SD. D: The expression of E-cadherin, Vimentin, Ki67, and YAP1 was analyzed by IHC in Lv-anti-SNHG16 NC group, Lv-anti-SNHG16 group, and Lvanti-SNHG16 + LV-Oe-YAP1 group. Error bars, SD. E: Representative images of CTC isolated from two mice, respectively. Scale bar, $20 \mu \mathrm{m}$. F: The MCTC ratio of mouse blood collected from Lv-anti-SNHG16 NC group, Lv-anti-SNHG16 group, and Lv-anti-SNHG16 + LvOe-YAP1 group. G-H: Representative images of metastatic lesions in the liver of mice in the Lv-anti-SNHG16 NC group, and representative images of metastatic lesions in the lung of mice in the Lv-anti-SNHG16 + Lv-Oe-YAP1 group. Representative hematoxylin and eosin-stained sections of metastatic nodules in liver and lung are shown. Scale bar, 200x. I: A schematic diagram illustrated the mechanism by which LncRNA SNHG16 promoted CRC progression and liver metastasis.

\section{Supplementary Files}

This is a list of supplementary files associated with this preprint. Click to download.

- TableS1.docx 
- FigureS1.tiff

- Figures2.tif

- Figures3.tif

- Figures4.tif 